\title{
Flight Performance Maneuver Planning for NASA's X-57 "Maxwell" Flight Demonstrator - Part 1: Power-Off Glides
}

\author{
Nicholas K. Borer, ${ }^{1}$ David E. Cox ${ }^{2}$ \\ NASA Langley Research Center, Hampton, Virginia, 23681, USA \\ Ryan D. Wallace ${ }^{3}$ \\ NASA Armstrong Flight Research Center, Edwards, California, 93523, USA
}

\begin{abstract}
Distributed Electric Propulsion technology is expected to yield up to a fivefold increase in high-speed cruise efficiency for NASA's X-57 "Maxwell" flight demonstrator when compared to a combustion-powered general aviation baseline. A portion of this increased efficiency is due to beneficial aero-propulsive interaction inherent to the distributed propulsion architecture. The measure of the relative increase in efficiency between a conventional and distributed propulsion wing will be extracted from comparisons between flight test data from the electrically powered X-57 Mod II configuration with a conventional wing, and from the electrically powered X-57 Mod III/IV configuration with a distributed propulsion wing. Flight test maneuvers that accommodate errors in instrumentation and the flight test environment are developed to establish the power-off drag characteristics for all X-57 configurations. Analysis of these maneuvers with typical errors, including pilot-in-the-loop simulation data that incorporates simulated atmospheric turbulence effects, shows that the proposed poweroff flight maneuvers can generate accurate power-off drag predictions for the X-57. These predictions show that the power-off differences in aerodynamic performance between the conventional and distributed propulsion configurations can be accurately measured from flight test data in the presence of typical data error sources.
\end{abstract}

\section{Nomenclature}

$a_{x} \quad=$ acceleration along aircraft body-frame $\mathrm{x}$-axis

$a_{y} \quad=$ acceleration along aircraft body-frame $y$-axis

$a_{z} \quad=$ acceleration along aircraft body-frame z-axis

$C_{D} \quad=$ coefficient of drag

$C_{L} \quad=$ coefficient of lift

$D=$ power-off drag force, relative to aircraft wind axes

$D E P=$ Distributed Electric Propulsion

$K_{i} \quad=$ polynomial coefficients in $\operatorname{drag} \operatorname{model}(i=0,1$, or 2$)$

$g_{0} \quad=$ acceleration due to gravity, typically $\sim 9.81 \mathrm{~m} / \mathrm{s}^{2}$

$h=$ starting altitude for selected performance maneuver, MSL

$I M U=$ Inertial Measurement Unit

$K C A S=$ knots calibrated airspeed

$K E A S=$ knots equivalent airspeed

KTAS = knots true airspeed

$L \quad=$ lift force, relative to aircraft wind axes

$m \quad=$ aircraft mass

$M R E \quad=$ Model Representation Error

$M S E \quad=$ Mean Squared Error

$M S L \quad=$ Mean Sea Level

${ }^{1}$ Aerospace Engineer, Aeronautics Systems Analysis Branch, MS 442, AIAA Senior Member.

${ }^{2}$ Senior Research Engineer, Dynamic Systems and Controls Branch, MS 308.

${ }^{3}$ Aerospace Engineer, Dynamics and Controls Branch, AFRC MS 4840-D, AIAA Member. 


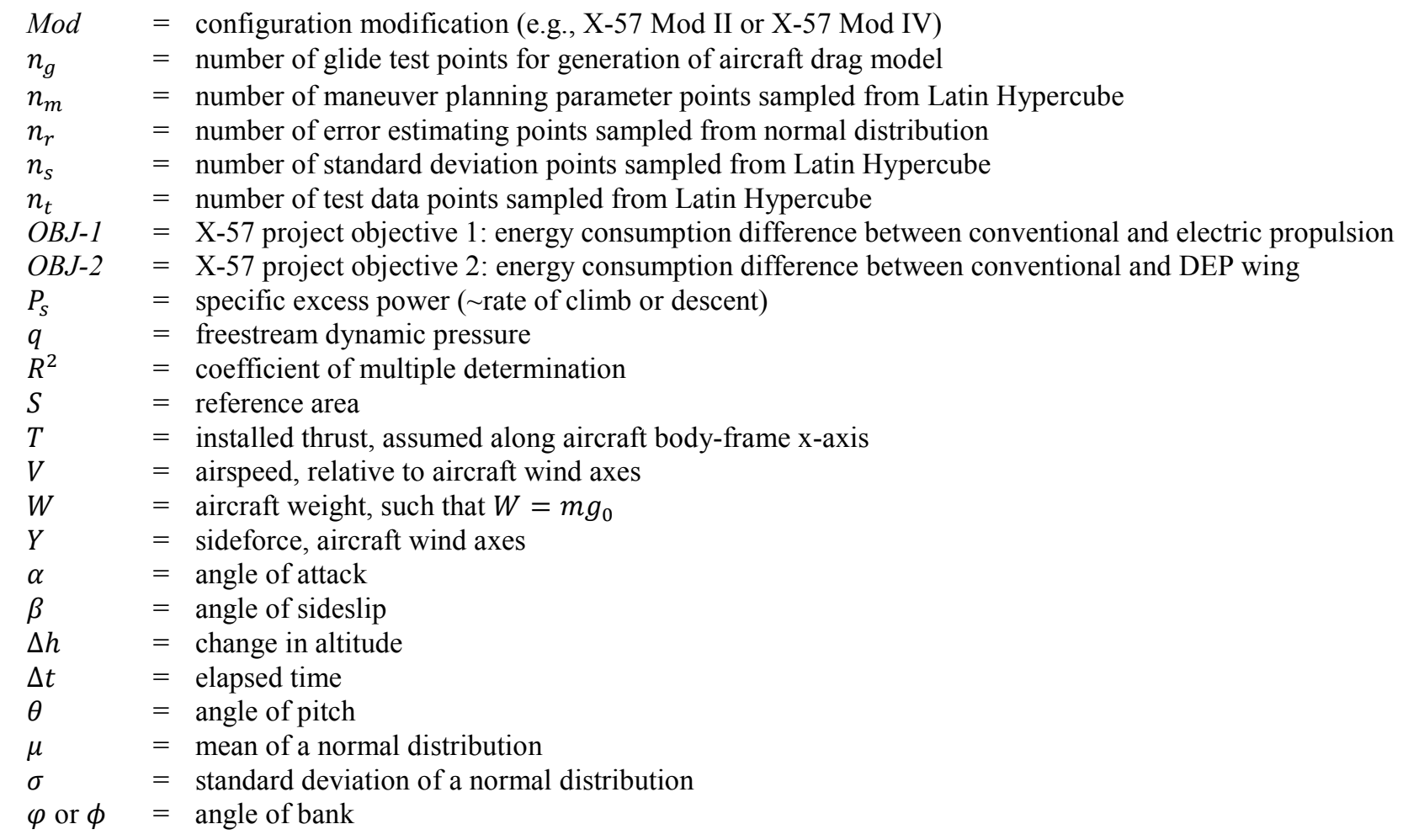

\section{Introduction}

$\mathrm{N}$ ASA's X-57 "Maxwell" is a flight demonstrator for Distributed Electric Propulsion (DEP) technology. This technology results from the confluence of distributed propulsion (the integration of propulsive devices strategically placed about the airframe to yield aero-propulsive benefits), and electric propulsion (the use of electric machines to drive propulsive devices). DEP technology is expected to yield a nearly fivefold reduction in onboard energy consumption on the X-57 in high-speed cruise flight [1], compared to the donor Tecnam P2006T [2], a conventionally fueled, combustion-powered aircraft that has a fuselage, empennage, and landing gear in common with the X-57.

The fivefold reduction target associated with DEP on the X-57 is apportioned between the use of electric propulsion (compared to the gasoline engine on the $\mathrm{P} 2006 \mathrm{~T}$ ) and the reduction associated with distributed propulsion (the aero-propulsive benefits of the X-57 distributed propulsion wing compared to the P2006T wing). These are referred to in the top-level X-57 project's objectives as OBJ-1 and OBJ-2, respectively [3]. The minimum success criteria associated with these objectives are as follows:

- X-57 OBJ-1: Consume less than 33\% of the energy of a baseline General Aviation (GA) aircraft at high-speed cruise.

- X-57 OBJ-2: Consume less than $83 \%$ of the energy of an electric General Aviation (GA) aircraft with a conventional wing at high-speed cruise.

An appropriate "high-speed cruise" target was explored in [1], and is defined in the X-57 Performance and Sizing requirements [4] as steady, level flight at 150 knots true airspeed (KTAS) at 8,000 ft above Mean Sea Level (MSL) in the 1976 U.S. Standard Atmosphere [5].

One may note that a fivefold reduction in energy requires an aircraft that consumes only $20 \%$ of the energy of the general aviation baseline, whereas the combined effects of OBJ-1 and OBJ-2 result in an aircraft that consumes approximately $0.33 \times 0.83=27 \%$ of the baseline energy. However, these are the project objectives from which all system requirements are derived and therefore represent the minimum achievable standard for project success. The project goal is a loftier, combined $20 \%$ energy consumption metric compared to the baseline. Estimates provided at the X-57 critical design review showed that the current distributed propulsion wing might result in an energy consumption that is $65 \%$ of that with a conventional wing, exceeding the requirement in OBJ-2 [6].

If the propulsion system has negligible effects on the aerodynamics of the aircraft, X-57 OBJ-1 can largely be inferred through ground tests. That is, if the power requirements at the high-speed cruise point are known for either the combustion-powered or electric variant, then a ground test can be used to measure the efficiency of the electric 
powertrain at this power setting vs. the efficiency of the combustion engine (as long as any altitude effects on power setting and efficiency are duplicated in the ground test environment). This approach will be used by the X-57 project to determine compliance with OBJ-1. However, determination of the aero-propulsive benefit of distributed propulsion effects requires operation in flight or powered wind tunnel environments to ensure that the interacting aero-propulsive effects are properly captured.

The X-57 project is planning a series of flight tests in different configurations that enable capture of flight data in both a baseline electric and DEP configuration. This paper describes the first of two major categories of flight test maneuvers planned to measure the X-57 performance. The first set of maneuvers, described in this paper, is focused on scrutiny of the power-off glide performance of the X-57, which is used to construct a parametric, power-off drag polar. A future paper will describe the approach used in power-on flight, in which the drag polar generated from the power-off glides will be used to complete assessment of OBJ-2.

This paper is organized to describe the analysis of the impact of different in-flight maneuver campaigns, measurements, and tolerances on the estimation of power-off performance characteristics of X-57 Mod II and later X$57 \mathrm{Mod}$ III/IV for determination of OBJ-2. Section III describes two different methods that will be used to estimate power-off aerodynamic forces from flight data. Section IV describes the analysis approach used to estimate the impact of variation in planning parameters and noise/error sources. Section V compares the results of the computational experiments of the two different methods within the maneuver planning tradespace and estimated error boundaries.

\section{A. X-57 Spiral Development Approach}

Demonstration of new technologies can be a challenging endeavor and requires a systematic approach to manage risk. The X-57 project uses a "spiral development" approach, which involves several different configuration modifications, or "Mods," as shown in Fig. 1. These "Mods" serve as interim demonstrations to reduce risk by testing different aspects of new hardware, software, and/or flight phenomena sequentially, rather than all at once.

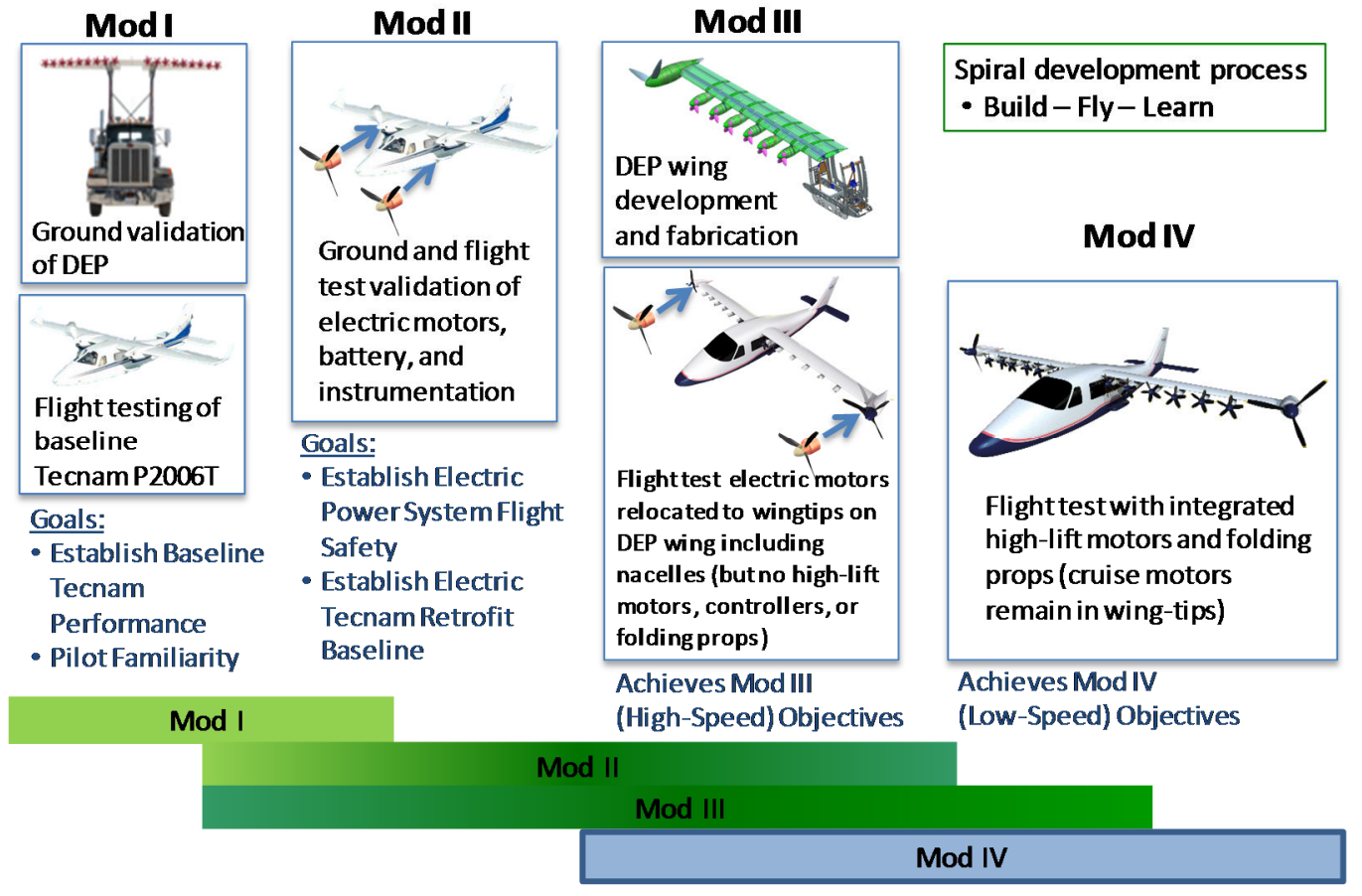

Fig. 1 X-57 spiral development through multiple "Mods" [1].

Mod I tests, completed in 2015, established the baseline, gasoline-powered aircraft performance. Mod II replaces the two gasoline engines on the stock Tecnam P2006T with electric motors and installs the X-57 high-voltage energy storage system in the aft cabin and cargo area. This Mod verifies the flight readiness of the primary traction electric propulsion system. Mod III replaces the large P2006T wing with a much smaller wing suitable for DEP and moves the cruise motors tested on Mod II out to the wingtips. This configuration determines the high-speed cruise efficiency, including aerodynamic and aero-propulsive benefits. Mod IV determines how the low-speed performance with 12 additional high-lift motors and propellers compares to the original aircraft. In practice, Mods III and IV will be the same aircraft; however, Mod III flights will be made with the high-lift propeller system deactivated. 
X-57 OBJ-2 can be determined by comparing the flight data from X-57 Mod II with flight data from X-57 Mod III. The X-57 instrumentation includes detailed information on the current and voltage associated with the traction electrical bus, which determines the power consumption at any given setting. However, it is generally impossible to perfectly match test conditions between flights, so performance models of the X-57 Mod II and Mod III will be created to help normalize conditions for later comparison. This is standard practice for aircraft performance comparison; it is otherwise essentially impossible to directly compare flight data between two different flight tests without a tightly controlled environment (e.g., wind tunnel). These performance models will come from two types of maneuvers: Power-Off Glides, and Power-On Sweeps. The methods used for data reduction for Power-Off Glides are described below, and the methods used for Power-On Sweeps will be the subject of a future paper.

\section{Power-Off Performance Estimation}

Two methods are considered for evaluation of power-off performance. These use different measurement and estimation techniques, and the data produced by these methods are subject to different error sources. These are referred to as Method 1 and Method 2 for this paper.

\section{A. Power-Off Glides, Method 1}

The purpose of the power-off glide maneuver is to estimate power-off, trimmed drag characteristics for verification of performance data. This drag model will also be used to estimate net thrust observed during the Power-on Sweeps. The basis for drag estimation in Power-Off Glides comes from the basic equation for unaccelerated, steady specific excess power:

$$
P_{S}=\frac{(T-D) V}{W}
$$

where $P_{S}$ is the specific excess power (generalized as rate of climb or rate of descent), $T$ is the thrust (assumed to be aligned with the aircraft body x-axis), $D$ is the drag, $V$ is the true airspeed, and $W$ is the aircraft weight. For this estimate to hold, lift is assumed to be essentially equal to weight (unaccelerated flight, small angles).

In a true power-off glide, the thrust is set to zero. In a quasi-steady descent, where the airspeed and descent rates are nearly constant, the power-off drag can be estimated from:

$$
D=-W \frac{\Delta h / \Delta t}{V}
$$

where $\Delta h$ is the change in altitude and $\Delta t$ is the change in time during the glide.

This very simple approach enables the estimation of drag with minimal instrumentation - all of the necessary data can be gathered by the pilot using only cockpit instrumentation. Even with a sophisticated instrumentation package, as will be present on the X-57, this technique enables a very simple, repeatable procedure to be used by the test pilot when gathering the data. The procedure is as follows:

- Set target airspeed and configuration at sufficient altitude to allow recovery. Set the aircraft into a zerothrust configuration, and trim for glide.

- When descent airspeed and rate are stabilized, note altitude and time (or start timer). Pilot maintains airspeed as closely as possible.

- When maneuver is completed, note final altitude and elapsed time. Recover for next maneuver or trim for another data point.

In some aircraft, establishing a zero-thrust configuration can be a challenge. Most single-engine aircraft do not have the ability to "feather" the propeller into a minimum drag configuration, so windmilling drag and other windmilling propeller effects can occur near low or idle power settings that introduce noise and bias into the drag estimate. Many multi-engine aircraft, such as the Tecnam P2006T, do have the ability to feather propellers, so the drag of the propellers can be minimized. However, operational and safety reasons ${ }^{*}$ often preclude operators of combustion-powered aircraft from feathering all propellers. Because of the electric propulsion system, the X-57 Mod II, III, and IV configurations will not suffer from these considerations.

\footnotetext{
${ }^{*}$ In-flight shutdowns of internal combustion engines can cause rapid, uneven cooling that can reduce operational lifetime and increase maintenance issues. Furthermore, restarting internal combustion engines in flight with this uneven cooling can be challenging, which is why intentional shutdowns of all engines are rare in flight tests.
} 
This approach is susceptible to flight perturbations, particularly turbulence, wind shear, and other atmospheric effects. Variations in the altitude, airspeed, and/or elapsed time measurements can also introduce error. The effectiveness of this technique in the presence of such variations is estimated in subsequent sections of this paper.

\section{B. Power-Off Glides, Method 2}

The X-57 will have a high-fidelity instrumentation package to measure a number of flight test parameters, including time-tagged inertial and air data. As such, these data can be used to estimate aerodynamic forces in a glide, in a way that accounts for perturbations in the atmosphere and flight test techniques. If the aircraft is represented as a rigid body with six degrees of freedom, the equations of motion along the body axes are as follows:

$$
\begin{aligned}
& a_{x} m=T-Y \sin \beta+L \cos \beta \sin \alpha-g_{0} m \sin \theta-D \cos \alpha \cos \beta \\
& a_{y} m=Y \cos \beta-D \cos \alpha \sin \beta+g_{0} m \cos \theta \sin \phi \\
& a_{z} m=-D \cos \beta \sin \alpha-L \cos \alpha \cos \beta+g_{0} m \cos \theta \cos \phi
\end{aligned}
$$

where $a_{x}, a_{y}$, and $a_{z}$ denote the body force accelerations, $L$ is the vehicle lift and $Y$ is the vehicle sideforce expressed in the wind axis, $\alpha$ is the angle of attack, $\beta$ is the angle of sideslip, $\theta$ is the aircraft pitch angle, $\varphi$ is the aircraft bank angle, $m$ is the aircraft mass, and $g_{0}$ is the acceleration due to gravity, such that $W=m g_{0}$. These values are shown in Fig. 2 in terms of the aircraft body axes $x, y$, and $z$. A more detailed derivation can be found in Ref. [7].

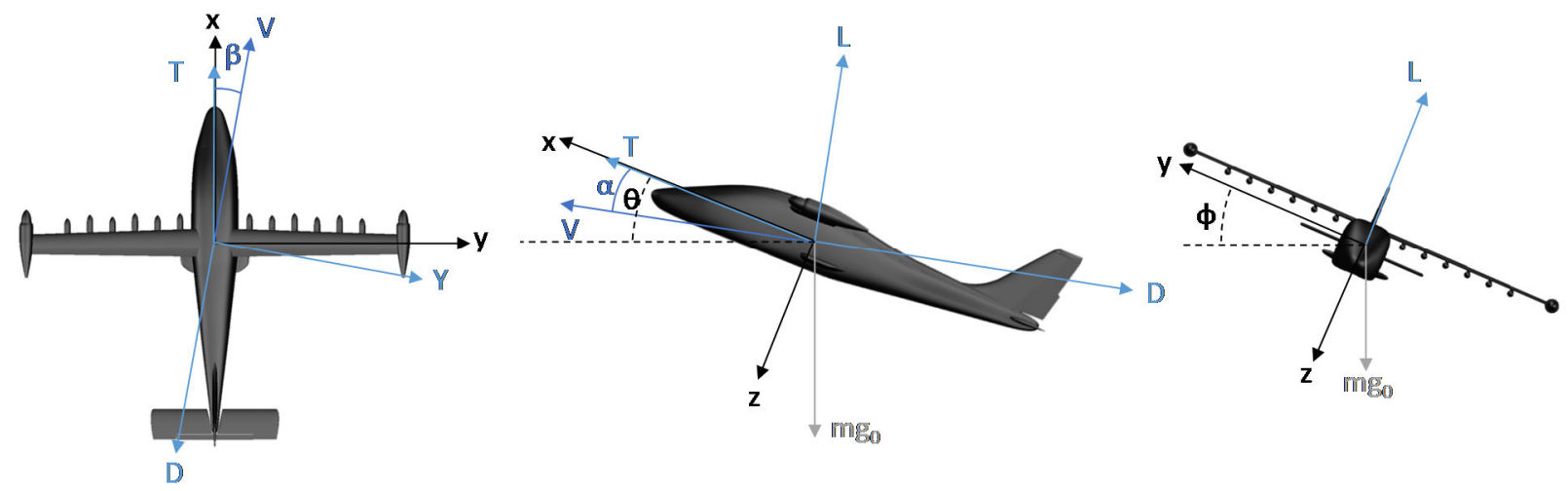

Fig. 2 Aircraft orientation in wind and body axes. Left: top view. Middle: side view. Right: front view.

The X-57 instrumentation package includes estimates for the aircraft Euler angles, including $\theta$ and $\phi$, body accelerations $a_{x}, a_{y}$, and $a_{z}$, and air data parameters, including $\alpha$ and $\beta$. The aircraft weight will be known at the start of the flight and will not appreciably change (a significant difference from combustion-powered aircraft that burn fuel over the course of the mission). As such, the quantity $m g_{0}$ will be known if the aircraft is weighed prior to the mission, and local values for $g_{0}$ can be approximated as constant. In such a case, during a power-off glide, where $T=0$, Eqs. (3-5) have only three unknowns: the aerodynamic forces $D, L$, and $Y$. Solving for these ${ }^{\dagger}$ values yields the following:

$$
\begin{aligned}
D= & -\frac{a_{x} m+g_{0} m \sin \theta+\frac{\sin \alpha\left(a_{z} m-g_{0} m \cos \phi \cos \theta\right)}{\cos \alpha}+\frac{\sin \beta\left(a_{y} m-g_{0} m \cos \theta \sin \phi\right)}{\cos \beta}}{\cos \alpha \cos \beta+\frac{\cos \beta \sin \alpha^{2}}{\cos \alpha}+\frac{\cos \alpha \sin \beta^{2}}{\cos \beta}} \\
L= & \frac{\frac{\cos \beta \sin \alpha\left(a_{x} m+g_{0} m \sin \theta+\frac{\sin \alpha\left(a_{z} m-g_{0} m \cos \phi \cos \theta\right)}{\cos \alpha}+\frac{\sin \beta\left(a_{y} m-g_{0} m \cos \theta \sin \phi\right)}{\cos \beta}\right)}{\cos \alpha \cos \beta+\frac{\cos \beta \sin \alpha^{2}}{\cos \alpha}+\frac{\cos \alpha \sin \beta^{2}}{\cos \beta}}-a_{z} m+g_{0} m \cos \phi \cos \theta}{\cos \alpha \cos \beta}
\end{aligned}
$$

\footnotetext{
$\dagger$ The MATLAB ${ }^{\circledR}$ Symbolic Math Toolbox [8] was used for this exercise, which may result in some unreduced terms.
} 


$$
Y=-\frac{\frac{\cos \alpha \sin \beta\left(a_{x} m+g_{0} m \sin \theta+\frac{\sin \alpha\left(a_{z} m-g_{0} m \cos \phi \cos \theta\right)}{\cos \alpha}+\frac{\sin \beta\left(a_{y} m-g_{0} m \cos \theta \sin \phi\right)}{\cos \beta}\right)}{\cos \alpha \cos \beta+\frac{\cos \beta \sin \alpha^{2}}{\cos \alpha}+\frac{\cos \alpha \sin \beta^{2}}{\cos \beta}}-a_{y} m+g_{0} m \cos \theta \sin \phi}{\cos \beta}
$$

Eqs. (6-8) show that the aerodynamic forces can be directly observed from synchronized data in the instrumentation package. If the errors in the accelerations, Euler angle estimates, and air data are sufficiently low, this may be a more robust estimation of drag than can be derived from the simplified approach based on timed glides. The effectiveness of this technique in the presence of typical variations is estimated in subsequent sections of this paper.

\section{Analysis Approach}

The salient challenge of computing flight performance metrics from models extracted from flight test data lies in developing an approach that yields credible results despite the presence of error in the test and modeling techniques. A number of techniques were used to evaluate the impact of error on the measurement of the test data. These error estimates were then propagated to understand the impact of the errors on the performance metrics in the modeling technique used to generalize the data from the test points into estimates of aircraft performance over a specified flight envelope. As no actual flight data exists, simulation techniques were used to create synthetic "truth" data to test the techniques.

\section{A. Test Point Error Estimation}

As noted in the previous section, two methods will be used for power-off performance characterization during X57 flight testing. These methods are subject to different error sources. A summary of the methods used to evaluate the impact of these errors is described below.

\section{Error Analysis for Power-Off Glides, Method 1}

"Method 1" is one approach to estimate the aerodynamic drag of the aircraft in the power-off configuration. Per Eq. (2), there are four possible error sources: errors in $W, \Delta h, \Delta t$, and $V$. Given that this measurement occurs over a finite time period, and Eq. (2) assumes that the values remain constant, these errors can result from bias in the measurement as well as time-varying error due to variations in the atmosphere or pilot control technique. As a first estimate to aid in the flight test maneuvers, the errors are assumed to be constant, randomly selected biases in the particular measurement timeframe. In such a case, three fundamental questions emerge:

a) If a given persistent, random bias error exists in the measurements of $W, \Delta h, \Delta t$, and $V$, what is the sensitivity of drag measurement to parametric variations in the maneuver planning parameters: $V$, starting altitude $h, \Delta h$, and $W$ ?

b) For a particular set of maneuver planning parameters $V, h, \Delta h$, and $W$, what is the sensitivity of drag measurement to variation of the bias errors in the measurements of $W, \Delta h, \Delta t$, and $V$ ?

c) What is the effect of pilot technique and changing environment on these measurements?

Questions (a) and (b) are answered using "truth" performance data derived from the data generated during design of the X-57, as summarized in Refs. [1], [6], and [9]. Question (c) is answered using "truth" data derived from the simulation experiments described in the subsection below. In all cases, these values of "truth" data are used not only to help plan the maneuvers, but also to provide a surrogate for developing the data reduction approach that will be used during actual flight tests.

(a) Variation in Maneuver Planning Parameters-For (a), a parametric range of values is considered for $V, h, \Delta h$, and $W$. The aircraft drag is estimated as a simple average of the drag from the "truth" dataset at the starting and ending altitudes, with this value of "truth" used as $D$ in Eq. (2) to solve for the "truth" $\Delta t$. An error estimator is added to the "truth" values of $W, \Delta h, \Delta t$, and $V$ measured in these maneuvers. For this case, MATLAB ${ }^{\mathbb{B}}$ 's randn function is used to generate the error estimate. The randn function generates a pseudorandom number in a normal distribution from an input standard deviation, denoted by $\sigma$. For these experiments, $\sigma$ is held constant, while $n_{r}$ pseudorandom numbers help generate error statistics in a Monte Carlo-type simulation.

To answer question (a), the experimental design space must be sampled, meaning that error statistics have to be generated for a sample of flight maneuver planning parameter points $V, h, \Delta h$, and $W$. For this analysis, Latin Hypercube sampling, in the form of MATLAB ${ }^{\mathbb{R}}$ s 1 hsdesign function, is used to sample $n_{m}$ maneuver planning points. This function samples these planning parameters in a space-filling, pseudorandom pattern to provide a uniform sampling of the space. "Truth" data is created for the particular $V, h, \Delta h$, and $W$ sampled from the Latin Hypercube. The resulting "truth" measurements of $W, \Delta h, \Delta t$, and $V$ are perturbed by $n_{r}$ error estimators. For each of these $n_{r}$ 
points, the resulting $D$ is calculated from Eq. (2) using the "measured" rather than "truth" data. The error statistics are then developed for each "truth" point vs. the $n_{r}$ measured points. The mean and sigma error estimates are derived by applying the MATLAB ${ }^{\circledR}$ mean and std functions, respectively, to the $n_{r}$ population set.

(b) Variation in Maneuver Error Statistics-Question (b) is resolved similarly and is slightly more straightforward. "Truth" data exists only at a single, user-specified maneuver planning parameter point $V, h, \Delta h$, and $W$. Now, Latin Hypercubes are used to sample a space of $n_{s}$ values of $\sigma$ for the measured $W, \Delta h, \Delta t$, and $V$. Then for each sampled $\sigma$ set, $n_{r}$ error estimators are selected from a uniform normal distribution using the same procedure as for question (a). For each of the $n_{s} \sigma$ points, the $n_{r}$ error estimators are aggregated into a single average mean and standard distribution of error.

(c) Variation in Pilot Technique and Flight Environment-The final set of variations tested for "Method 1" include variations in the test data introduced by atmospheric turbulence and pilot technique. The atmospheric turbulence models are simply settings used in the simulation approaches that include "none," "light," "moderate," and "severe," as described in a later section. The pilot technique was measured by using actual pilot-in-the-loop performance in the piloted simulation in these different turbulence environments, with a small subset of different initial conditions. The pilots were instructed to glide for a particular amount of time or altitude loss, and then either change airspeed to another value, or recover to level flight.

\section{Error Analysis for Power-Off Glides, Method 2}

Method 2 relies on data from the measured body axis linear accelerations $\left(a_{x}, a_{y}, a_{z}\right)$, two Euler angles $(\theta, \phi)$, and two air data parameters $(\alpha, \beta)$. The linear accelerations and Euler angles will be estimated from a multi-axis Inertial Measurement Unit (IMU) mounted in the X-57 instrumentation package, which provides filtered estimates of these accelerations, angles, and angular rates. The air data angles will be measured by vanes on a long research air data probe located in the nose of the aircraft.

The instrumentation error models vary based on the type of measurement. The linear accelerations are directly computed from the accelerometers in the IMU, which exhibit bias, scale factor, and noise errors. The Euler angles are part of a filtered estimate from integration of the IMU data (which includes inputs from accelerometers, gyroscopes, and magnetometers) and errors are generally given as static and dynamic accuracy at the $95 \%(2-\sigma)$ confidence level. Similarly, the air data sensors provide filtered estimates that are calibrated for installation error on the initial X-57 flights, with errors expressed at the $95 \%(2-\sigma)$ confidence level. The error estimates used for this study are as follows:

- Air data probe angles $(\alpha, \beta): \pm 0.5^{\circ}(2-\sigma$ project calibration goal $)$

- Pitch and roll angles $(\theta, \phi): \pm 0.15^{\circ}(2-\sigma$, adapted from [10])

- Linear accelerometer noise $\left(a_{x}, a_{y}, a_{z}\right): \pm 0.003 g(1-\sigma$, adapted from [10])

- Linear accelerometer bias $\left(a_{x}, a_{y}, a_{z}\right): \pm 0.005 g(2-\sigma$, adapted from [10])

- Linear accelerometer scale factor $\left(a_{x}, a_{y}, a_{z}\right): \pm 0.06 \%$ (2- $\sigma$, adapted from [10])

The Method 2 error estimates are derived by Latin Hypercube sampling of the parameter space of $\alpha, \beta, \theta, \phi, a_{x}$, $a_{y}$, and $a_{z}$ to again achieve a pseudorandom uniform collection of test data points, denoted by $n_{t}$. Eqs. (6-8) are applied to each sample point to generate "truth" data. A pseudorandom normal distribution is used to generate $n_{r}$ error estimators for each of the $n_{t}$ test data points, to represent the random noise present in the seven parameters. The linear accelerations are then modified by $n_{b}$ pseudorandom normal bias errors, and further modified by $n_{f}$ pseudorandom normal scale factor errors. In total, this requires evaluation of Eqs. (6-8) on $n_{t} \cdot n_{r} \cdot n_{b} \cdot n_{f}$ total samples to compute the difference from the $n_{t}$ "truth" samples. The results are largely plotted as histograms and include the estimation of mean and standard deviation of error estimates.

\section{B. Model Error Estimation}

In general, aircraft drag is a strong, predictable function of lift coefficient and dynamic pressure [11]. Other variables, such as aircraft configuration (e.g., landing gear and flap position), airspeed, and local atmospheric conditions have a predictable effect as well. OBJ-1 and OBJ-2 are for a specific configuration (cruise), with a specified altitude and speed. Nevertheless, for generality, the experiments for X-57 will include a range of speeds and altitudes. The variations in these speeds and altitudes will be such that the strongest influence on the aircraft drag will be related to the lift coefficient and dynamic pressure. The lift and drag coefficients are given as

$$
\begin{gathered}
C_{L}=L / q S \\
C_{D}=D / q S,
\end{gathered}
$$


where $C_{L}$ is the lift coefficient, $C_{D}$ is the drag coefficient, $q$ is the dynamic pressure, and $S$ is the reference area, usually related to the projected wing planform area. In the case of the X-57, the reference area for Mod I and II is $14.76 \mathrm{~m}^{2}$, and for Mod III/IV it is $6.194 \mathrm{~m}^{2}$. In the speed range and configuration of interest to the X-57, $C_{D}$ can typically be fit as a quadratic function of $C_{L}$, such that:

$$
C_{D}=K_{0}+K_{1} C_{L}+K_{2} C_{L}^{2}
$$

where $K_{0}, K_{1}$, and $K_{2}$ are polynomial coefficients to be computed. For the X-57, these will be found through a leastsquares regression of data points that relate the drag estimated from the power-off glide maneuvers to the lift. For Method 1, the lift can be inferred from the aircraft weight (the load factor for this maneuver is approximately unity), the drag can be estimated per Eq. (2), and the dynamic pressure estimated from the air data instrumentation. ${ }^{\ddagger}$ For Method 2, lift and drag are directly calculated from the accelerations, Euler angles, and air data, with the potential for far more data points. In the current flight test plan, data from Method 1 will be used to generate the drag polar shown in Eq. (11); the data from Method 2 is planned mostly as a possible means to verify the drag polar.

The model presented in Eq. (11) requires only three unique test points to develop a least-squares model, but additional data can provide an estimate of the error associated with the fit model. However, additional glide data can be a challenge to gather in flight tests, so the number of data points selected is a careful balance between the model error (favors more test points) and test efficiency (favors fewer test points). To help determine the appropriate set of $n_{g}$ glide test points, simulated "truth" data was fit to Eq. (11) with various error estimators. These error estimators were sampled from a normal random distribution in a Monte Carlo simulation, again using MATLAB ${ }^{\mathbb{B}}$ 's randn function with a fixed input $\sigma$. A total of $n_{r}$ random trials were conducted for each of the $n_{g}$ points within a glide maneuver candidate set. A linear least-squares model was fit for each of the $n_{r}$ trials, and model fit error statistics were generated for each fit model; namely, the Mean-Squared Error (MSE) and coefficient of multiple determination $\left(R^{2}\right)$ [12]. The MSE and $R^{2}$ values were averaged over all of the $n_{r}$ trials to determine the impact of the different experimental designs for the glides as well as the impact of different assumptions in the error in each test point.

However, these statistics only speak to how well the model fits the data in the dataset and do not necessarily indicate how well the models fit the entire domain. This Model Representation Error (MRE) was found by comparing the fit models from each of the $n_{r}$ trials to "truth" data generated from the simulation dataset for 51 different lift coefficients, resulting from variation in airspeed from 81-135 KCAS in 1-knot increments at 6,000 ft MSL on a standard day. The resulting differences were collected into a mean and a standard deviation to understand the error trends for the models for a given experimental design and error assumption within each data point.

\section{Generation of "Truth" Data from Simulations}

\section{Desktop ("Batch") Simulation}

A desktop engineering ("batch") simulation was developed in MATLAB ${ }^{\circledR} /$ Simulink $^{\circledR}$ for flight dynamics analysis and for conducting batch-level simulations. The model can be setup to represent the X-57 vehicle in each of the four configurations outlined in Fig. 1. Data for the mass and geometry properties in each configuration is well-defined and drawn from detailed CAD models of each vehicle configuration. The aerodynamic database for X-57 Mod IV is still under development, but baseline models exist, combining results of wind tunnel testing of an unpowered model with CFD and lower-order codes for blown lift increments.

The results presented in this paper are generated for the Mod II configuration, which has very minor outer mold line changes from the stock Tecnam aircraft. Some aerodynamic parameters, therefore, are available as published estimates from the aircraft manufacturer [13]. In addition, data for this airframe was obtained from flight tests conducted on an instrumented Tecnam P2006T with conventional engines in Mod I. The resulting model does not attempt to capture stall effects but provides a database that includes control powers, rate derivatives, and configuration dependence of the flap and landing gear systems.

In addition to the aerodynamic database, a propulsion model was developed for the Mod II vehicle configuration. Blade element analysis of the three-bladed propellers was used to generate the coefficients of power and torque over a range of blade pitch angles. A propeller pitch controller is implemented in the simulation to track RPM commands, subject to the bandwidth limits of the electrically actuated controller. This model provides both propulsive response to torque and estimates of drag from the propellers when they are unpowered and windmilling.

\footnotetext{
* This information can also be derived from the aircraft's airspeed indicator, corrected for installation error and
} nonstandard atmospheric conditions, if known. 
For the batch results used in this paper, the desktop simulation was trimmed to an equilibrium flight condition, solving for the control surface inputs, wind angles, and glide slope that provide for zero acceleration and zero rate response on the vehicle. Simulations are run from this point with stick-fixed inputs to hold the same flight condition, with modest drift due to density changes in the atmosphere model. These unpowered glide simulations provide an ideal source of trajectory data for estimating drag coefficients. In total, three glides were used to test the methods developed in this paper:

- Glide 1: 8,000 ft MSL starting altitude, trimmed to zero thrust at $135 \mathrm{KEAS}, 40 \mathrm{~s}$ duration

- Glide 2: 6,891 $\mathrm{ft}$ MSL starting altitude, trimmed to zero thrust at $120 \mathrm{KEAS}, 40 \mathrm{~s}$ duration

- Glide 3: 6,074 ft MSL starting altitude, trimmed to zero thrust at $85 \mathrm{KEAS}, 40 \mathrm{~s}$ duration

A more realistic set of simulations, including atmospheric turbulence and its effect on the gliding trajectory, were also run. Turbulence enters the simulation as a time-dependent perturbation of the wind angles. The frequency content in this disturbance is consistent with the Dryden turbulence model, as described in Appendix-A of MIL-STD-1797A [14]. The same glides, starting from the same trim conditions, were used with turbulence selected as "moderate."

\section{Piloted Simulation}

The X-57 piloted simulator employs the Core 6-DOF nonlinear simulation, version 9.01, used at NASA's Armstrong Flight Research Center (AFRC) as the basic simulation framework. Written in C++ and JAVA, the AFRC's Core simulation provides an adaptable platform for integration of specific aerodynamic, propulsion, and other project unique models. The estimated mass properties of the Mod II and Mod III/IV research aircraft as well as the aerodynamic and propulsion models from the $\mathrm{MATLAB}^{\circledR} /$ Simulink $^{\circledR}$ desktop simulation, described above, are integrated into the AFRC's Core simulation by autocoding of the desktop simulation models into C++. Included in the AFRC Core simulation are atmospheric models for winds, gusts, and turbulence. The AFRC Core simulation uses the Dryden turbulence model, referenced above, to output turbulent wind velocities as function of height above ground level, true airspeed, and severity. The X-57 piloted simulator runs on an Oracle X4-4 server that drives the out-thewindow displays and simulation cockpit hardware/software. Representative aircraft hardware such as switches, pilot controls, and gauges are incorporated in the simulator's cockpit to give a realistic cockpit layout to the pilots.

To provide a representative set of flight data, X-57 test pilots performed a series of glides in the Mod II piloted simulator. The pilot initiated the piloted simulation run by obtaining a power-on trim with flaps and gear up at an altitude between 8,500 and 9,000 ft MSL, ensuring sufficient height above ground to obtain the desired glide maneuvers. Once power-on trim was established, the pilot then pulled both throttles back to idle, initiating the glide. During the glide, the pilot performed three zero-thrust trim shots at various constant airspeeds for the duration of approximately 40 seconds. In addition to performing the glide runs in quiescent atmosphere, the pilot also conducted runs in low and moderate severity turbulent air. Performing the glides in turbulent air introduced unsteadiness into the analysis, which may impact the accuracy of drag estimates using Method 1. In addition to varying the atmospheric conditions, the pilots also explored initializing the glides in an off-condition setting. For the off-condition, the pilot initiated the glide before the constant speed propellers were fully in the feathered position, causing a drag force transient during the first part of the glide. This was used as an example of variation in pilot technique.

\section{Results of Computational Experiments}

Several computational trials were used to help identify the impact of error on the development of X-57 performance models. This included analysis of the error sources for the individual test points, analysis of the experimental designs used to select the total number of test points, and the impact of pilot-in-the-loop test technique and external environment on overall model error. Unless otherwise noted, these error estimates follow from the techniques outlined in the section above.

\section{A. Test Point Error}

\section{Power-Off Glides, Method 1}

The estimation of test point error associated with the variation in maneuver planning parameters used the approach outlined in section IV.A.1(a). The bounds of the flight test envelope for the power-off drag maneuvers were sampled from the following parameters with a Latin Hypercube having $n_{m}=100$ :

- $\quad V: 80$ to $140 \mathrm{KCAS}$

- $h: 4,000$ to $10,000 \mathrm{ft} \mathrm{MSL}$

- $\Delta h:-200$ to $-1200 \mathrm{ft}$

- $\quad W: 2,800$ to $3,100 \mathrm{lbf}$ 
Fairly coarse 1- $\sigma$ error values for the measurements, taken to be representative of what could occur over the maneuver with little more than primary flight instrumentation, were estimated as follows, with $n_{r}=400$ :

- $\quad W: 20 \mathrm{lbf}$

- $\Delta h: 25 \mathrm{ft}$

- $\Delta t: 0.2 \mathrm{~s}$

- $\quad V: 2 \mathrm{KCAS}$

The drag, time in glide, $\%$ mean change in drag estimate, and \% 1- $\sigma$ error of drag estimate statistics were gathered from a total of 40,000 data points, corresponding to 400 pseudorandom trials, each of 100 "truth" data points sampled from the Latin Hypercube. In this case, the "truth" data was derived from a known drag polar, representative of the drag polar used in the simulations. The resulting mean error in the drag estimates was essentially zero, as expected, since the measurement error estimates were centered on the "truth" data. Histograms of the responses for the 1- $\sigma$ error in the 100 drag estimates and the estimated time for each glide (to meet the specified $\Delta h$ ) are show in Fig. 3.
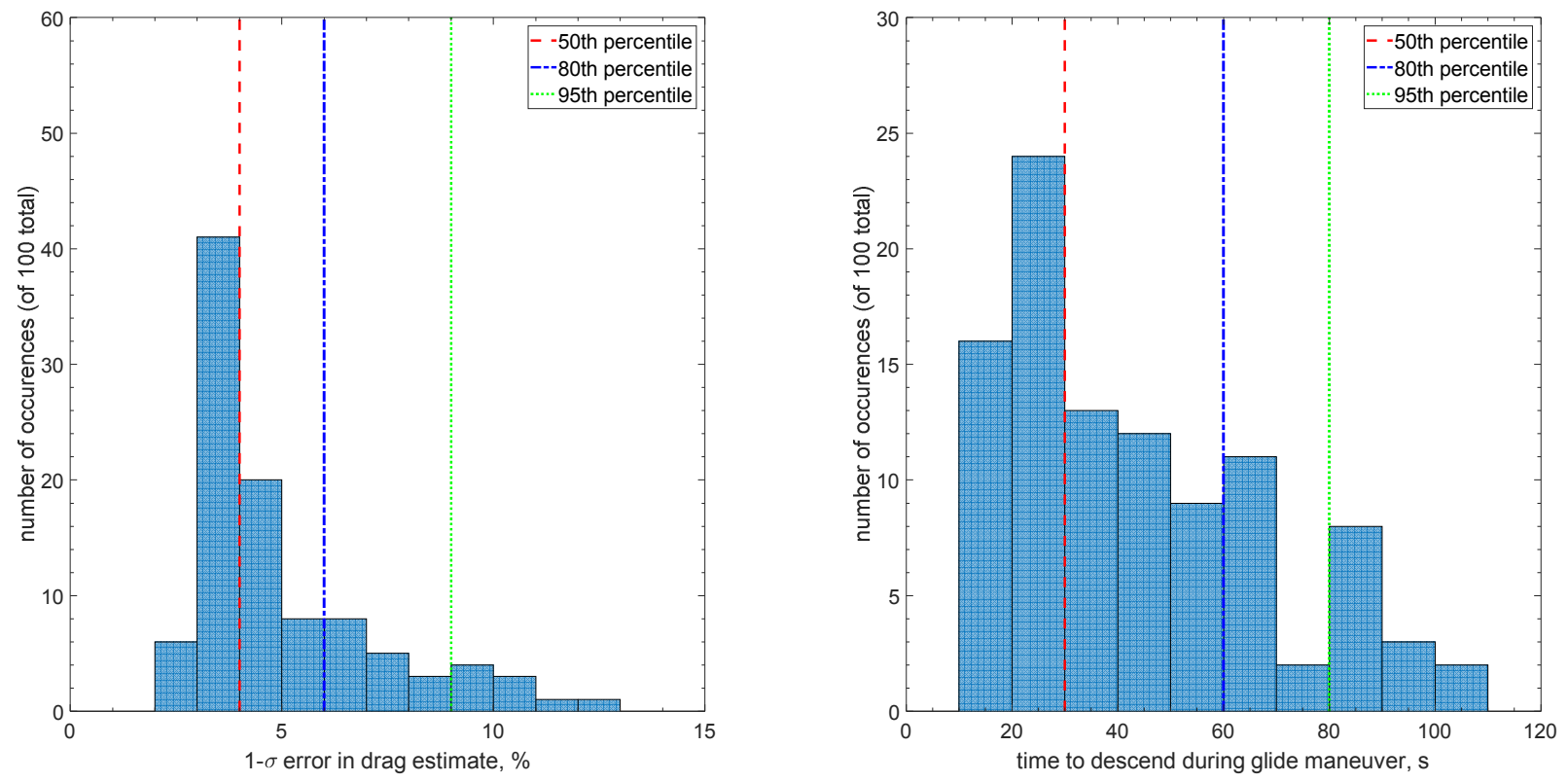

Fig. 3 Estimated statistics in glide operational envelope. Left: error in drag estimate. Right: time to descend.

To illustrate any dependence of maneuver design on error, the error, change in time, and drag were fit to a full quadratic response surface. The resulting prediction profiler from $\mathrm{MATLAB}^{\mathbb{E}}$ 's rstool displays instantaneous, interactive partial derivatives from the currently selected input values. The 1- $\sigma$ errors were found to be lower for maneuvers with more altitude change, with diminishing returns found for glides with over $700 \mathrm{ft}$ of altitude loss. Higher speeds tended to change the shape of the partial derivative associated with altitude loss to favor less error with greater altitude loss. This indicates that time at test point, rather than amount of altitude loss, may be a more important metric for maneuver planning. Overall, single-point drag errors of less than 5\% were observed for all glides except those with the smallest values of $\Delta h$.

Next, per the approach outlined in section IV.A.1(b), the variations in test point error with change in measurement error statistics were evaluated for the fixed test condition of $V=90 \mathrm{KCAS}, h=8,000 \mathrm{ft} \mathrm{MSL}, \Delta h=-500 \mathrm{ft}$, and $W=$ 2,900 lbf. Figure 4 shows the 1- $\sigma$ drag error estimates for this particular glide maneuver based on a Latin Hypercube design with the following $1-\sigma$ variations in measurements:

- $\quad W: 5-25 \mathrm{lbf}$

- $\Delta h: 5-25 \mathrm{ft}$

- $\Delta t: 0.05$ to $0.5 \mathrm{~s}$

- $\quad V: 0.5-3 \mathrm{KCAS}$

For this particular flight condition, $1-\sigma$ drag errors were generally within $5 \%$ for all but the highest error settings. Overall, this provides some confidence that the error in drag prediction during glides using the Method 1 approach can generally be kept to within 5\%, and possibly within 3\%, for all but the shortest glide maneuvers. However, variations in pilot technique and the flight test environment will add to these errors, as seen below. 


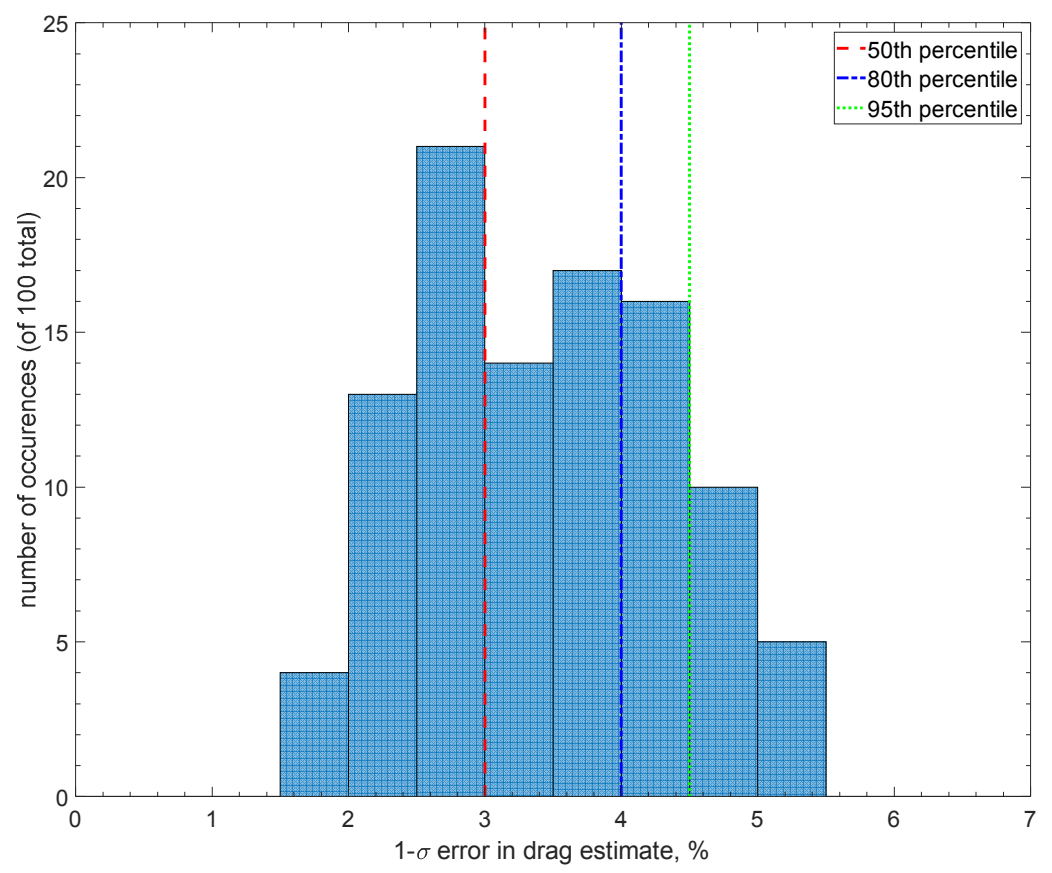

Fig. 4 Drag estimate error based on variation of error in measurement parameters for nominal maneuver.

2. Power-Off Glides, Method 2

The drag estimation for Method 2 needs to be valid over an appropriate range of measured body-axis linear accelerations $\left(a_{x}, a_{y}, a_{z}\right)$, Euler angles $(\theta, \varphi)$, and air data parameters $(\alpha, \beta)$. The variation in these parameters was estimated based on data from the piloted simulation glide maneuvers with the largest turbulence variation ("moderate"). The resulting changes in the relevant air data angles, Euler angles, and linear accelerations from this piloted simulation run are shown in Fig. 5. Slightly narrower ranges were used to uniformly sample the space with a Latin Hypercube consisting of $n_{t}=100$ points:
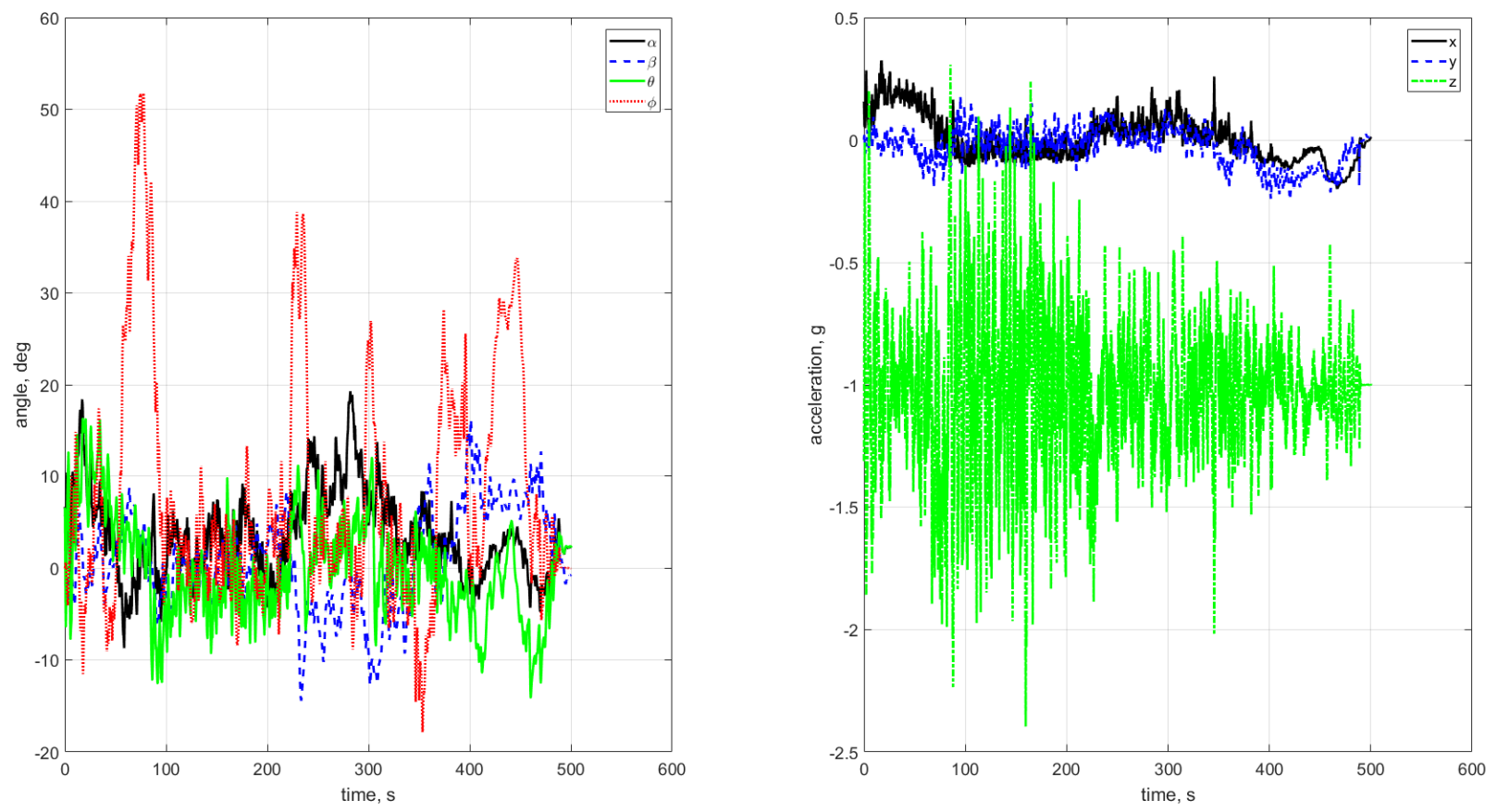

Fig. 5 Variations seen in $\alpha, \beta, \theta, \phi$, and linear accelerations during most turbulent piloted simulation. 
- $a_{x}, a_{y}:-0.3$ to $0.3 g$

- $a_{z}:-2.0$ to $0.3 \mathrm{~g}$

- $\alpha, \beta, \theta:-15^{\circ}$ to $15^{\circ}$

- $\phi:-30^{\circ}$ to $30^{\circ}$

The error estimates associated with Method 2, discussed in section IV.A.2, were generated from pseudorandom normal distributions with $n_{r}=30, n_{b}=20$, and $n_{f}=20$. This resulted in 1.2 million drag estimates across a wide range of flight conditions and possible instrumentation errors. The resulting error distribution and cumulative distribution functions are shown in Fig. 6.
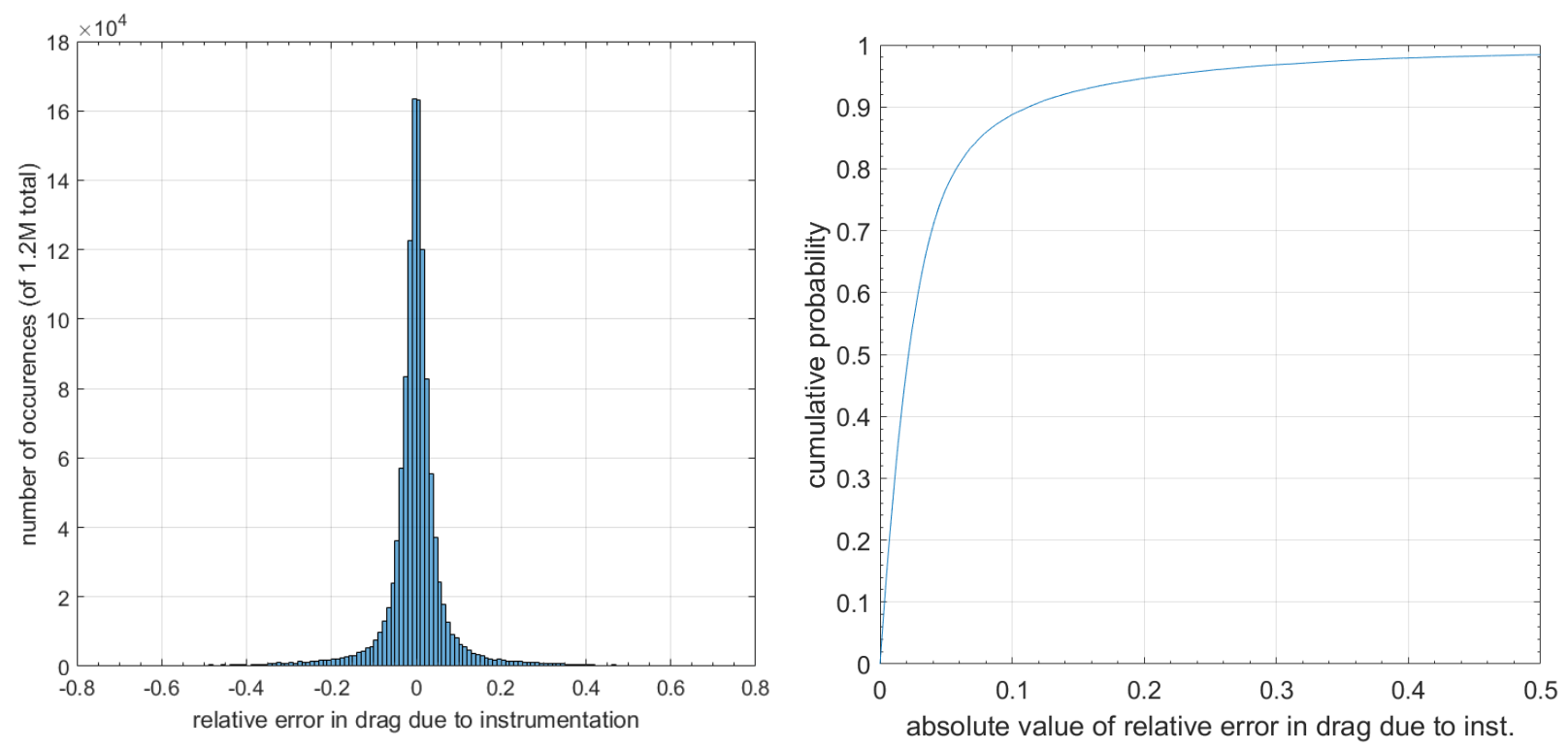

Fig. 6 "Method 2" Drag error estimates due to instrumentation errors. Left: probability density function of relative error. Right: cumulative probability of absolute value of relative error. Both distributions truncated due to "long tails" of relative error.

The relative drag error estimates for Method 2 with the simulated instrumentation errors shows a sharp peak but very wide "tails," resulting in a non-normal distribution. The cumulative probability shows that approximately $4 \%$ error exists at the $68 \%$ probability level, which is typically associated with $1-\sigma$. However, it also indicates $20 \%$ error at about the $95 \%$ cumulative probability level which is typically taken as $2-\sigma$. This indicates that while Method 2 may be a promising technique, the project plan of using Method 1 for flight test planning, with Method 2 as a backup approach, is likely appropriate. Still, further development may ultimately favor data generated from Method 2.

\section{B. Maneuver Design and Model Error}

The Test Point Error estimates form the basis of the Model Error Estimates. Method 1 is used as the basis for flight test planning. Therefore, flight at a minimum of three different lift coefficients (unaccelerated glides at a constant calibrated airspeed) is necessary to generate the model introduced in Eq. (11). Although more data points are always welcome, generation of this data on X-57 test flights will be challenging. The glide maneuver will require that the X57 pilots climb to an appropriate altitude and airspeed, shut off the motors, feather the propellers, and glide at the specified airspeed for a fixed amount of altitude loss or a fixed amount of time. Time permitting, the aircraft will be configured to the next glide speed. At some point, the X-57 will be in proximity to the ground, in which case the propellers will need to be unfeathered, and the motors restarted, to allow the flight test crew to adjust glideslope while approaching to land. ${ }^{\S}$ Two factors further exacerbate this challenge: (1) the X-57 has a fairly limited energy supply,

\footnotetext{
$\S$ One unique asset of the test range at NASA's Armstrong Flight Research Center is the availability of many long runways, and, if necessary, a dry lakebed to facilitate landing. For now, the X-57 project is developing its flight test plans with the assumption that hard-surfaced runways are to be used, with the lakebed or other sites only used for contingency operations. This paper assumes the airplane will need to be configured for powered flight at a suitable altitude to allow for typical airport traffic pattern operations.
} 
limiting the maximum altitude available to start the glides, and (2) the propeller pitch controller on the X-57 cruise motors operates very slowly, on the order of one degree per second. At a typical climb or cruise pitch condition, the propellers may take close to one minute to fully feather. Likewise, the propellers may take approximately one minute to return to the appropriate pitch position to allow for maneuvering flight in the airport traffic pattern. This bounds the useful glide maneuver envelope from a high of approximately 8,000 ft MSL to a low of approximately 5,000 ft MSL.

The models developed to meet OBJ-2 need only to be valid for cruise flight. For X-57 Mod II flight test planning, the drag polar only needs to range from an upper speed of approximately $135 \mathrm{KCAS}$ (near the high-speed cruise point used for OBJ-2 comparison) to no lower than the speed of the best rate of climb, which occurs near 85 KCAS. Hence, these are used as approximate bounds on the velocity of the glides.

Other operational factors are considered as well. The X-57 Mod II configuration will have the highest sink rate at the highest speeds within the aforementioned velocity envelope, and the lowest sink rates at the lower end of the envelope. Furthermore, the lower end of the velocity envelope is approximately the speed needed for typical traffic pattern operations. Therefore, it is beneficial from the safety of flight (reaction time close to ground) and operational point of view to have the fastest glides occur at the highest altitudes, with the slowest glides closest to the ground.

Considering all these factors, the maneuver designs investigated for the model error analysis in this paper assume that no more than three constant-speed glides are likely in a single flight. In addition, no more than three flights are assumed to be available for data collection. The resulting glide maneuvers used for the model error analysis consider the permutations shown in Table 1. Cases 1-3 include the same three data points, with 0,1 , and 2 repeated flights, respectively, and Case 4 considers two flights with six unique data points. The altitudes listed represent the average altitude of the glide used to estimate the "truth" drag data.

Table 1 Glide Maneuver Test Cases for Model Error Analysis

\begin{tabular}{l|l|l|l|l}
\hline & Case 1 & Case 2 & Case 3 & Case 4 \\
\hline Flight 1 & $135 \mathrm{KCAS} / 7,000 \mathrm{ft}$ & $135 \mathrm{KCAS} / 7,000 \mathrm{ft}$ & $135 \mathrm{KCAS} / 7,000 \mathrm{ft}$ & $135 \mathrm{KCAS} / 7,000 \mathrm{ft}$ \\
& $120 \mathrm{KCAS} / 6,000 \mathrm{ft}$ & $120 \mathrm{KCAS} / 6,000 \mathrm{ft}$ & $120 \mathrm{KCAS} / 6,000 \mathrm{ft}$ & $105 \mathrm{KCAS} / 6,000 \mathrm{ft}$ \\
& $85 \mathrm{KCAS} / 5,000 \mathrm{ft}$ & $85 \mathrm{KCAS} / 5,000 \mathrm{ft}$ & $85 \mathrm{KCAS} / 5,000 \mathrm{ft}$ & $85 \mathrm{KCAS} / 5,000 \mathrm{ft}$ \\
\hline Flight 2 & N/A & $135 \mathrm{KCAS} / 7,000 \mathrm{ft}$ & $135 \mathrm{KCAS} / 7,000 \mathrm{ft}$ & $125 \mathrm{KCAS} / 7,000 \mathrm{ft}$ \\
& & $120 \mathrm{KCAS} / 6,000 \mathrm{ft}$ & $120 \mathrm{KCAS} / 6,000 \mathrm{ft}$ & $115 \mathrm{KCAS} / 6,000 \mathrm{ft}$ \\
& & $85 \mathrm{KCAS} / 5,000 \mathrm{ft}$ & $85 \mathrm{KCAS} / 5,000 \mathrm{ft}$ & $95 \mathrm{KCAS} / 5,000 \mathrm{ft}$ \\
\hline Flight 3 & N/A & & $135 \mathrm{KCAS} / 7,000 \mathrm{ft}$ & N/A \\
& & & $120 \mathrm{KCAS} / 6,000 \mathrm{ft}$ & \\
& & & $85 \mathrm{KCAS} / 5,000 \mathrm{ft}$ & \\
\hline
\end{tabular}

Model fit statistics were gathered for each of these maneuver test cases using a least-squares fit of the drag coefficient to Eq. (11). The "truth" data for each flight in each case was varied to three different random error statistics - normally distributed, $1-\sigma$ errors in drag estimates of $3 \%, 5 \%$, and $10 \%$, respectively. These approximate the average drag error estimates found from Method 1 for the $50^{\text {th }}, 80^{\text {th }}$, and greater than $95^{\text {th }}$ percentiles, respectively. A total of 400 random error estimates were generated for each case, and the results compared at the three 1- $\sigma$ levels. The resulting model fit statistics, averaged for the 400 random fit cases, are given in Table 2. Note that these statistics relate to the fit of the data to the absolute $C_{D}$ in Eq. (11), not the percent error.

Table 2 Model Fit Error Statistics for Glide Maneuver Test Cases

\begin{tabular}{|c|c|c|c|c|c|c|c|c|}
\hline Case & \multicolumn{2}{|c|}{1 (3 points, 3 CLS) } & \multicolumn{2}{|c|}{2 (6 points, $\left.3 C_{L S}\right)$} & \multicolumn{2}{|c|}{3 (9 points, 3 CLS) } & \multicolumn{2}{|c|}{4 (6 points, $\left.6 C_{L S}\right)$} \\
\hline Metric & MSE & $\mathrm{R}^{2}$ & MSE & $\mathrm{R}^{2}$ & MSE & $\mathrm{R}^{2}$ & MSE & $\mathrm{R}^{2}$ \\
\hline$\sigma=3 \%$ & 0 & 1 & $2.19 \times 10^{-6}$ & 0.991 & $1.78 \times 10^{-6}$ & 0.988 & $1.79 \times 10^{-6}$ & 0.988 \\
\hline$\sigma=5 \%$ & 0 & 1 & $6.09 \times 10^{-6}$ & 0.976 & $4.95 \times 10^{-6}$ & 0.968 & $4.96 \times 10^{-6}$ & 0.966 \\
\hline$\sigma=10 \%$ & 0 & 1 & $2.44 \times 10^{-5}$ & 0.910 & $1.98 \times 10^{-5}$ & 0.881 & $1.99 \times 10^{-5}$ & 0.874 \\
\hline
\end{tabular}

As expected, the fully saturated design (Case 1) is always able to fit the data perfectly since there are no degrees of freedom for error (a minimum of three data points is required to fit the quadratic model). The fit statistics otherwise appear to be strong for the other cases as well, per these metrics. Still, model fit statistics rarely tell the full story; they just indicate how well the model can predict the points that were used to generate the model. As such, the model representation error was estimated per the technique discussed in section IV.B., which uses 51 "truth" data points. The resulting model representation error statistics are shown in Table 3. Here, mean model representation error $\mu_{M R E}$ and 
its standard deviation $\sigma_{M R E}$ refer to how well, on average, models fit to the three cases with the three different test point error distributions represented the 51 "truth" data cases.

Table 3 Model Representation Error Statistics for Glide Maneuver Test Cases

\begin{tabular}{|c|c|c|c|c|c|c|c|c|}
\hline Case & \multicolumn{2}{|c|}{1 (3 points, $\left.3 C_{L S}\right)$} & \multicolumn{2}{|c|}{2 (6 points, $\left.3 C_{L S}\right)$} & \multicolumn{2}{|c|}{3 (9 points, $\left.3 C_{L S}\right)$} & \multicolumn{2}{|c|}{4 (6 points, $\left.6 C_{L S}\right)$} \\
\hline Metric & $\mu_{M R E}$ & $\sigma_{M R E}$ & $\mu_{M R E}$ & $\sigma_{M R E}$ & $\mu_{M R E}$ & $\sigma_{M R E}$ & $\mu_{M R E}$ & $\sigma_{M R E}$ \\
\hline$\sigma=3 \%$ & $2.07 \%$ & $1.25 \%$ & $1.40 \%$ & $0.855 \%$ & $1.15 \%$ & $0.635 \%$ & $1.29 \%$ & $0.713 \%$ \\
\hline$\sigma=5 \%$ & $3.48 \%$ & $2.16 \%$ & $2.34 \%$ & $1.51 \%$ & $1.93 \%$ & $1.07 \%$ & $2.15 \%$ & $1.20 \%$ \\
\hline$\sigma=10 \%$ & $7.31 \%$ & $5.38 \%$ & $479 \%$ & $3.36 \%$ & $3.91 \%$ & $2.22 \%$ & $4.35 \%$ & $250 \%$ \\
\hline
\end{tabular}

These model representation error results help to inform the selection of the glide maneuver test cases. Other operational or safety considerations will ultimately be considered in addition to these estimates associated with the model error in response to test point error. Overall, it does appear that, if test point error can be limited to 1- $\sigma$ variations of less than $5 \%$ per test point, good fits with $2-\sigma$ average errors of less than $5 \%$ are possible for the aircraft drag characteristics.

\section{Flight Environment and Test Technique Error}

The final computational experiments involved the batch and piloted simulations to investigate the impact of atmospheric turbulence and pilot technique on the ability to gather test data for either Method 1 or Method 2. Initially, the batch simulation was used to verify that Method 2, with perfect input data, could faithfully represent the vehicle forces in glide. The "moderate" turbulence model was used, with the aircraft operated stick-fixed, trimmed per the initial conditions discussed in Section IV.C.1. An example of the resulting comparison is provided in Fig. 7 for the high-speed point, and the good match was typical of all of the forces and speeds evaluated. Given that Eqs. (6-8) are developed from the rigid body equations of motion, it is not surprising to see that Method 2 results in essentially identical values for aircraft drag compared to those used in the batch simulation.

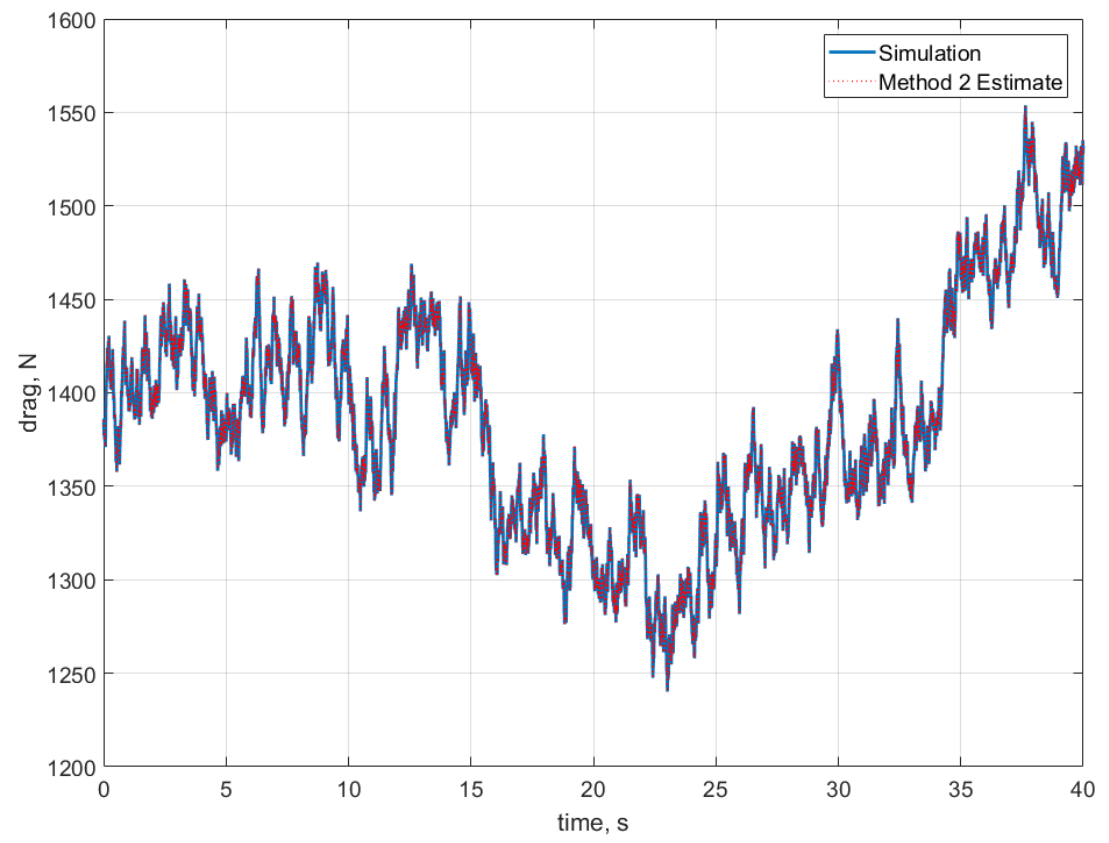

Fig. 7 Comparison of batch simulation drag ("truth") to drag derived from Method 2 (no additional error terms added) for $135 \mathrm{KCAS}$ glide with moderate turbulence.

The final set of computational experiments generated data from the piloted simulation. Five different flights were analyzed - two with no turbulence, two with low turbulence, and one with moderate turbulence. The flight plans loosely corresponded to the Flight 1 glide cases seen in Cases 1-3 in Table 1, except that the pilots were tasked to get on-condition, glide at the specified speed for 40 seconds, and then transition to the next speed. As noted in Section IV.C.2, the simulations were started at altitude, with different starting configurations (on-condition and off-condition). 
Drag estimation using Method 1 requires that the aircraft be held in a steady, unaccelerated glide for several seconds. As seen from Fig. 3, half the glides in the error evaluation matrix resulted in durations of 30 seconds or more. The statistical model fit from the previous exercise indicated that longer glides helped to reduce test point error. Though the maneuvers were planned to be flown by the pilots for a minimum of 40 seconds, an algorithm was developed to search through the simulation data (which is similar to the telemetry that will be recorded on actual X57 flights) to find glides that meet the requirements to be Method 1 data points with a fungible term to define the minimum time that met particular criteria to account for variations due to turbulence and pilot technique. These criteria included verifying that the telemetry indicated near-zero thrust (propellers fully feathered, propeller torque control set between -1 and $1 \mathrm{~N}-\mathrm{m}$ ) and airspeed variation of less than 2 KCAS over the duration of 30 seconds. For brevity, only three of the five piloted simulation cases are discussed. The other cases showed similar overall trends with turbulence.

The glide histories and associated Method 1 speed estimates are shown in Fig. 8 for a zero-turbulence case, and the associated drag coefficient estimates, derived from drag estimated from Eq. (2) and associated data from Eq. (10), are shown in Fig. 9 vs. the simulator "truth" data. The lift coefficient data in Fig. 9 is estimated from the aircraft weight and an assumed load factor of one, along with the averaged dynamic pressure per Eq. (9).
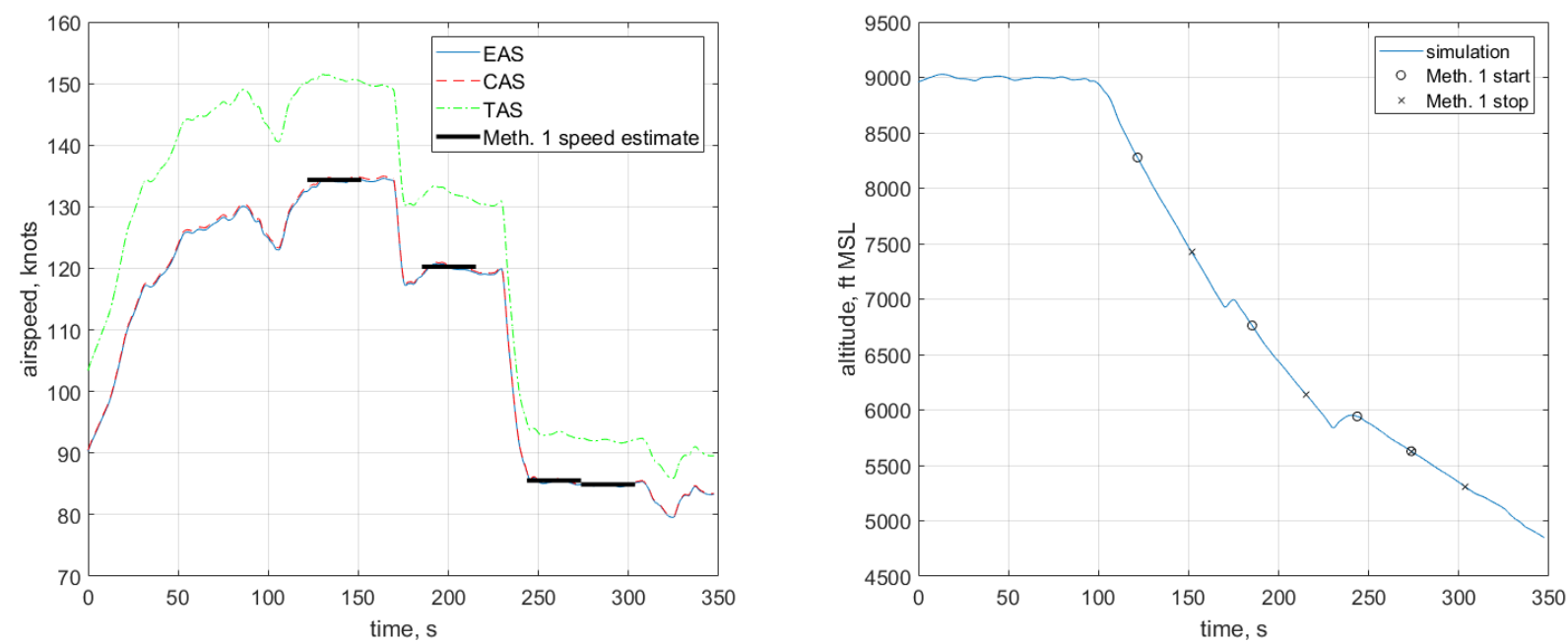

Fig. 8 Glide history for piloted simulation \#2 (starting from top-of-climb condition, no turbulence).
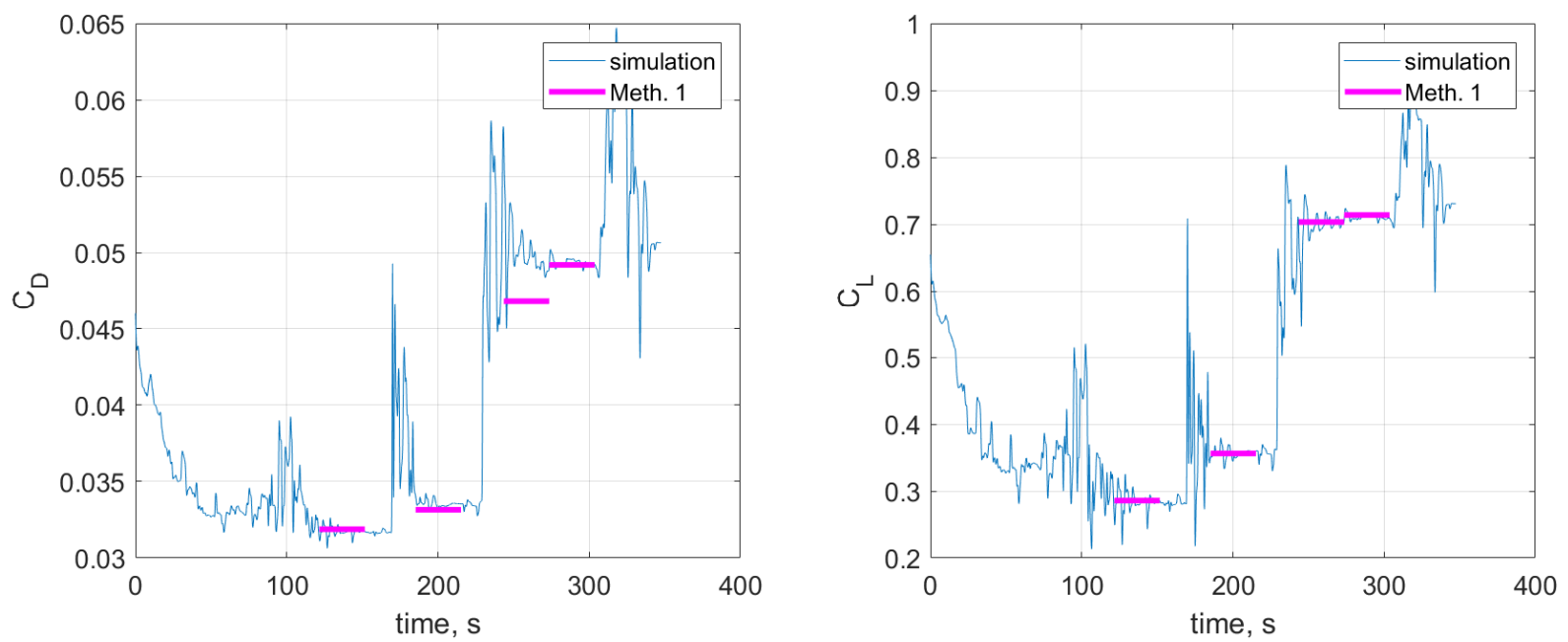

Fig. 9 Comparison of drag and associated lift coefficient estimates to truth data for piloted simulation \#2.

One of the challenges associated with Method 1 is that it requires flight in still air for the greatest accuracy. Atmospheric turbulence introduces updrafts and downdrafts that can interfere with the accuracy of Eq. (2), which is reliant on the rate of descent in still air. The glide history for one of the low turbulence cases is shown in Fig. 10, and Fig. 11 provides the resulting lift and drag estimates. 
In the first three simulation runs (\#1 not shown, $\# 2$ and $\# 3$ as shown in Figs. 8-11 above), it was possible to find at least three glide data points on each flight. For the flight with low turbulence, there was some difficulty meeting the speed tolerance for the final glide at the slowest airspeed and lowest altitude. In this case, the selected glide point was below 4,000 ft MSL, which is likely below the altitude of the lowest glide maneuver. The field elevation at the NASA Armstrong Flight Research Center is approximately 2,300 ft MSL. Note that the simulated flight in Fig. 10 appears to segue straight into an approach to land on the airfield given the altitude and velocity history. Still, the drag coefficient estimates for these flights match the admittedly shaky drag estimates well.

The simulation with moderate turbulence is shown in Fig. 12, with the resulting estimates for drag and lift coefficients shown in Fig. 13. In this case, there were no glides that met the criteria for Method 1 data points; that is, the pilot was unable to keep the tolerances in this turbulence condition. However, if the airspeed tolerance was kept to within $2 \mathrm{KCAS}$, but the time allowance was dropped from 30 seconds to 10 seconds, six possible data points appeared.
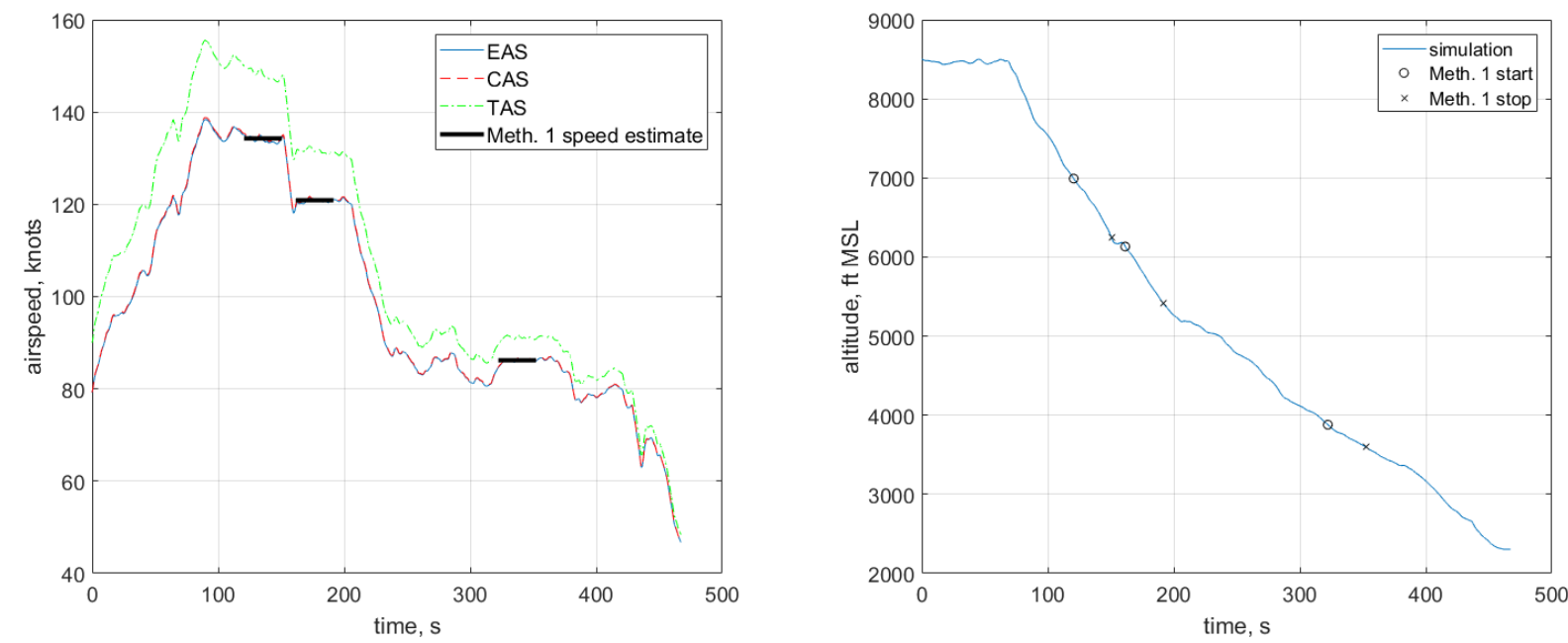

Fig. 10 Glide history for piloted simulation \#3 (starting from top-of-climb condition, low turbulence).
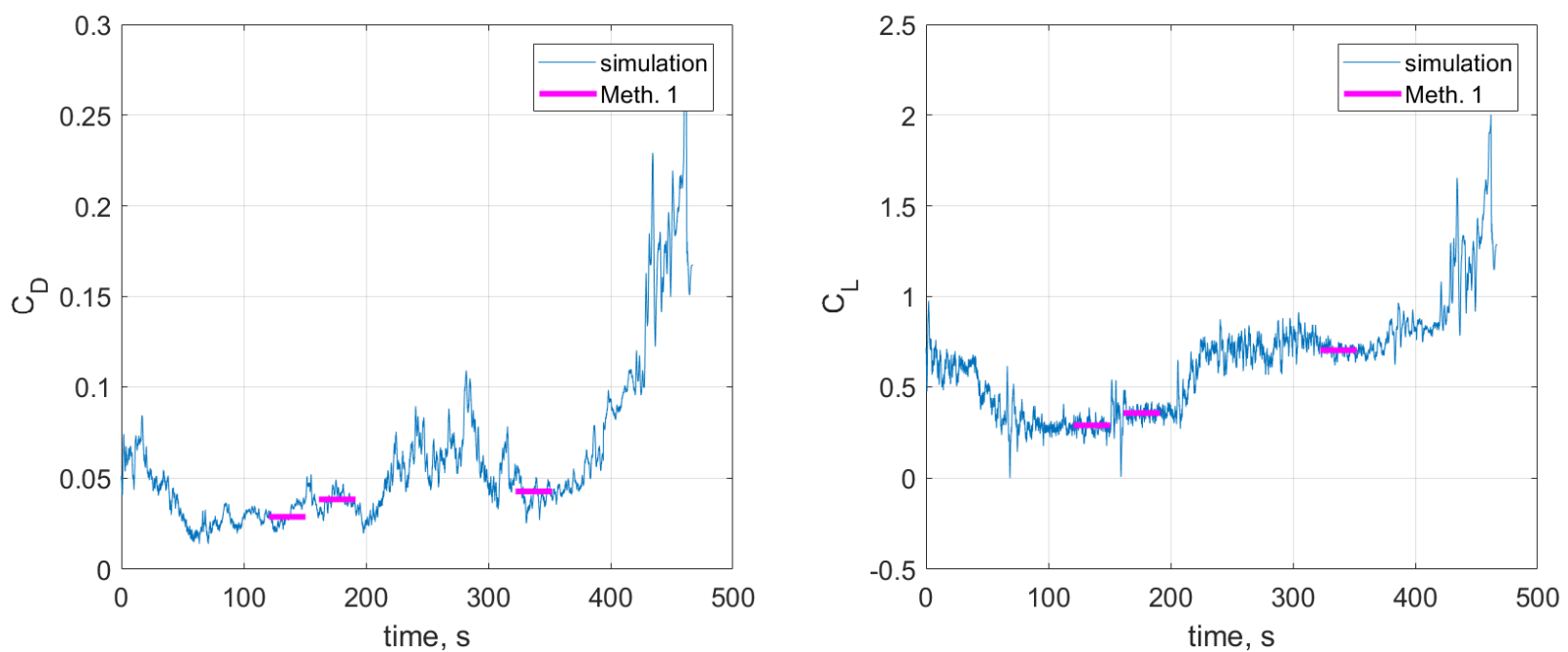

Fig. 11 Comparison of drag and associated lift coefficient estimates to truth data for piloted simulation \#3.

Though the Method $1 \mathrm{drag}$ and lift estimates from Fig. 13 appear to show appropriately averaged values for lift and drag, it is clear that the turbulence and associated pilot response to attempt to remain on condition result in violation of the provision for the maneuver to be conducted in unaccelerated flight. This is one of the main reasons 
the authors explored Method 2, which is not bound by such restrictions. Instead, the main restriction is a zero-thrust condition for Eqs. (6-8) to be valid.
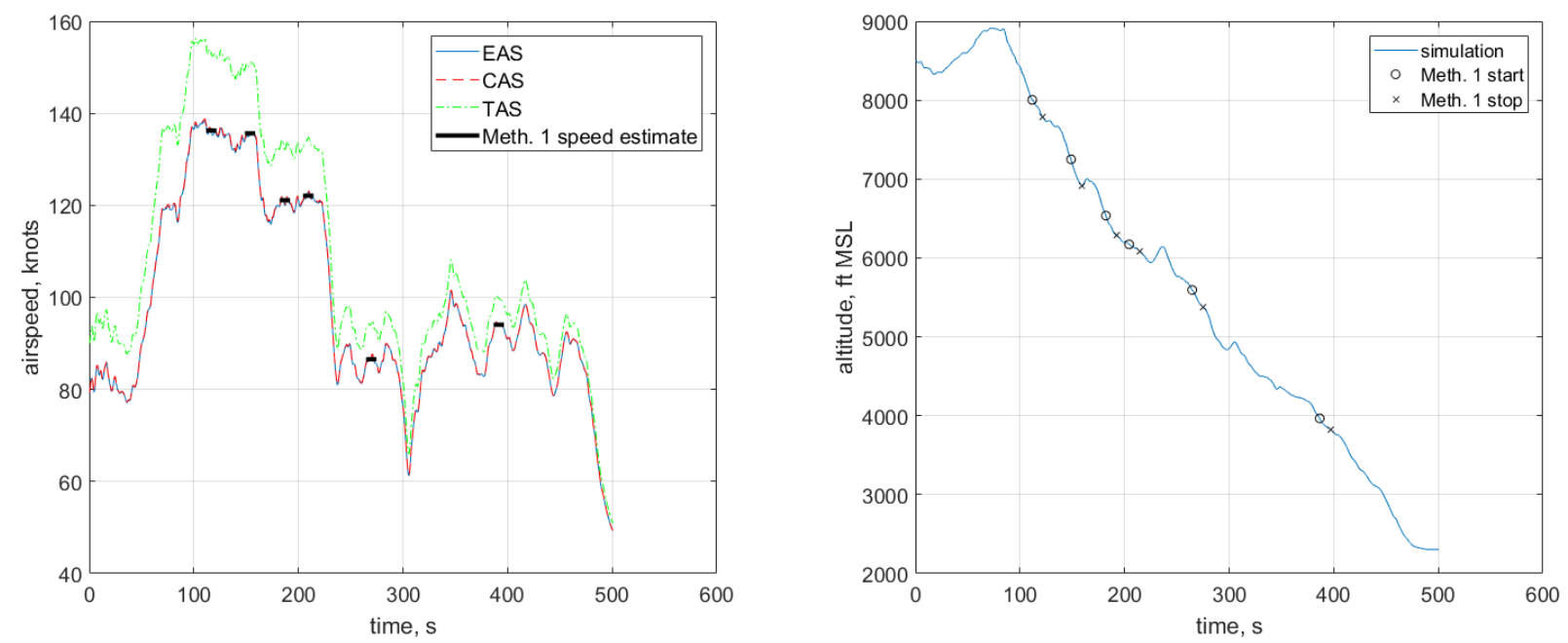

Fig. 12 Glide history for piloted simulation \#5 (starting from top-of-climb condition, moderate turbulence).
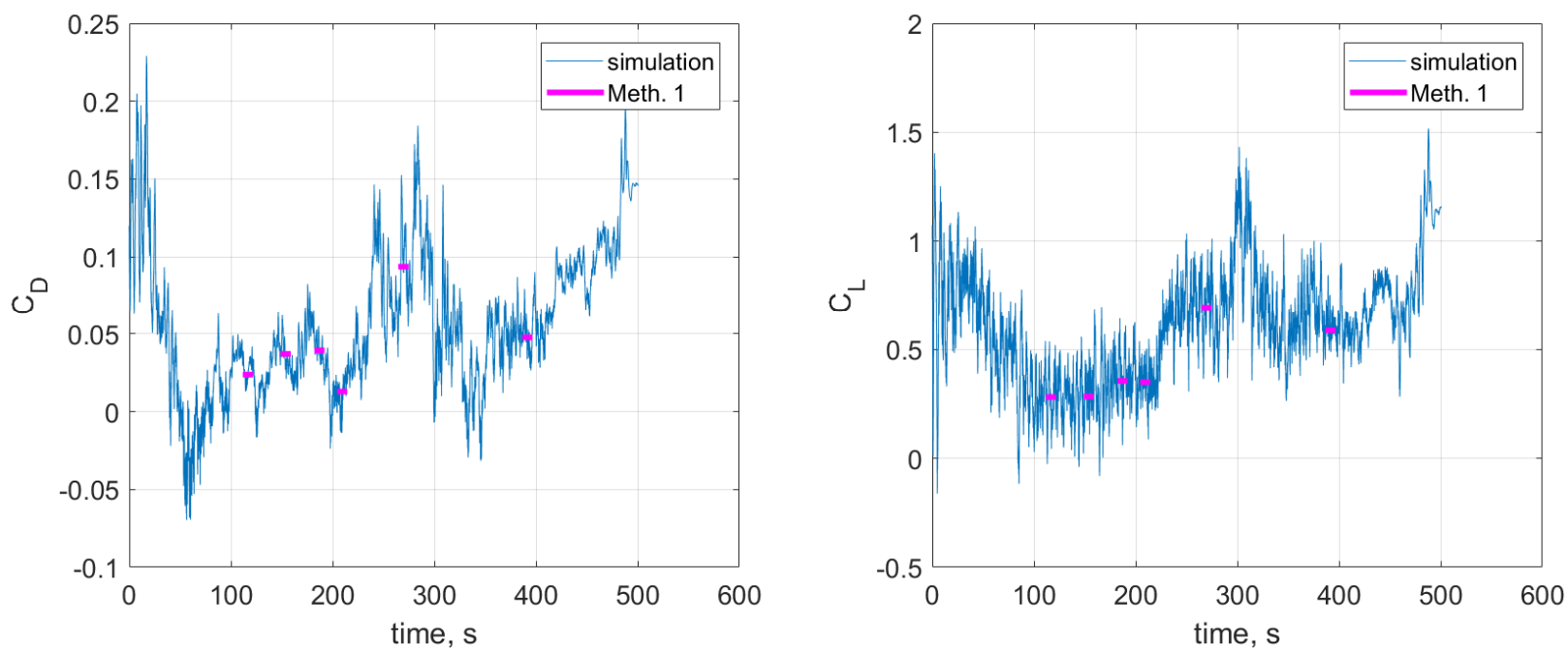

Fig. 13 Comparison of drag and associated lift coefficient estimates to truth data for piloted simulation \#5.

\section{Concluding Remarks}

One of the main objectives of the X-57 project is to demonstrate the change in aero-propulsive efficiency of the aircraft from its conventional Mod I and Mod II configurations to its cruise-efficient Mod III and Mod IV configurations. To understand the magnitude of this change in efficiency, the project team needs an accurate method to generate data in flight for the comparison between these configurations. A major part of the X-57's proposed flight data generation approach involves the use of power-off glides in flight. The X-57's electric propulsion system permits safe, sustained power-off glides with the propellers in the feathered position, minimizing windmilling effects for power-off drag estimation.

Two methods for estimating power-off aerodynamic drag of the X-57 were derived in this paper. Method 1, an approach used from the earliest days of flight, requires sustained, steady, unaccelerated flight in still air. During constant-speed glides, the resulting altitude loss and elapsed time can be used to estimate the power-off drag. Method 2, a more data-rich method appropriate for aircraft with more sophisticated sensors and data acquisition systems, involves the direct measurement of linear accelerations in the body axes, estimation of pitch and roll angles from an inertial measurement unit, and measurement of the angle of the relative wind to the aircraft body axes. In a true power- 
off glide, these data can be used to directly solve for the principal aerodynamic forces from the six-degree-of-freedom equations of rigid body motion. Given that this data is gathered at high rates, it is possible that Method 2 could be a very data-rich source of aerodynamic force estimation for the aircraft in a power-off glide.

A systematic series of computational experiments were conducted to evaluate the ability of Method 1 and Method 2 to generate data suitable for estimating a reasonable drag polar of the aircraft. Simulations were used to help characterize individual error sources, including: (1) errors in the individual test points from measurement errors, (2) errors introduced in the development of a model of the aerodynamic forces from this data, and (3) errors due to atmospheric effects and piloting technique. Initial estimates of the test point error showed that the average 1- $\sigma$ error used to gather data in Method 1 could generally be kept to 5-9\% of the "truth" drag estimate, depending on the flight condition and error in the measurements - though this assumed no other errors introduced dynamically into the glide maneuver. For Method 2, the resulting error distribution showed that $4 \%$ error in aerodynamic drag existed at the $68 \%$ confidence level; typically, a 1- $\sigma$ cumulative probability value. However, the resulting error distribution showed large errors at higher cumulative probabilities - error as high as $20 \%$ of drag at the $95 \%$ cumulative probability level over the ranges studied in this paper.

A total of four candidate flight test designs were scrutinized. The error analysis of the statistical model of the drag polar showed that normally distributed test point $1-\sigma$ errors of $5 \%$ could still result in an average 2- $\sigma$ error of approximately $5 \%$ for the entire drag polar with as few as six test points. This can reduce the number of required flights to as few as two dedicated power-off glide flights for the $\mathrm{X}-57$ project.

A series of batch and piloted simulations were used to estimate the impact of atmospheric variation and pilot technique on generation of data. The batch mode simulations confirmed that Method 2 could be used even in moderate turbulence to exactly recover the aerodynamic forces in the simulation, given perfect input data. The piloted simulations demonstrated that the data reduction techniques associated with Method 1 could yield reasonable estimates of power-off aerodynamic performance parameters in still air with variation in pilot technique. However, the random accelerations introduced for anything beyond low turbulence can quickly violate the steady flight provision associated with Method 1, and they made the pilot's job to maintain constant speed in a descent more difficult.

In summary, the results of this paper are being used to inform flight test planning for the power-off glide maneuvers used in the X-57 flight test campaigns across all different Mods. These maneuver plans also serve to train the pilots by using the piloted simulation prior to actual data collection flights, given that the test program may only have a few dozen flights across all Mods. In a future paper, the authors will explore the power-on maneuvers, as well as conduct some additional explorations of Method 2 in an effort to generate more data-rich approaches towards the development of X-57 performance models.

\section{Acknowledgments}

The authors thank NASA's Aeronautics Research Mission Directorate for their support of this effort. The X-57 project is currently funded under the Flight Demonstrations and Capabilities Project within the Integrated Aviation Systems Program, after being incubated in the Convergent Aeronautics Solutions Project within the Transformational Aeronautics Concepts Program. The authors also thank NASA pilots Timothy Williams and Wayne Ringelberg of the NASA Armstrong Flight Research Center for their assistance with generating the piloted simulation data used in this paper. Finally, the authors thank Ethan Nieman and Michael Frederick of the NASA Armstrong Flight Research Center for providing data on the X-57 inertial measurement unit and air data probe, respectively.

\section{References}

[1] Borer, N. K., Patterson, M. D., Viken, J. K., Moore, M. D., Clarke, S., Redifer, M., Christie, R., Stoll, A., Dubois, A, Bevirt, J., Gibson, A., Foster, T., Osterkamp, P., "Design and Performance of the NASA SCEPTOR Distributed Electric Propulsion Flight Demonstrator," $16^{\text {th }}$ AIAA Aviation Technology, Integration, and Operations Conference, AIAA AVIATION Forum, (AIAA 2016-3920), June 2016. doi: 10.2514/6.2016-3920

[2] Costruzioni Aeronautiche Tecnam S.r.1, "P2006T - Aircraft Flight Manual,” Doc. No. 2006/044, 4th Ed - Rev. 0, Italy, 2015.

[3] National Aeronautics and Space Administration, "X-57 Maxwell Objectives and Requirements Document (ORD)," ORDCEPT-002, Revision A, January 2018.

[4] National Aeronautics and Space Administration, "X-57 Maxwell Performance and Sizing Subsystem Requirements," REQCEPT-003, August 2016.

[5] National Aeronautics and Space Administration, "U.S. Standard Atmosphere, 1976," NASA-TM-X-74335, October 1976.

[6] National Aeronautics and Space Administration, "Scalable Convergent Electric Propulsion Technology and Operations Research (SCEPTOR) Critical Design Review: Day 2 Package," November 2016, available at https://www.nasa.gov/sites/default/files/atoms/files/sceptor_cdr_day_2 package.pdf, accessed 2 November 2018. 
[7] Etkin, B., Reid, L. D., Dynamics of Flight: Stability and Control, $3^{\text {rd }}$ Ed., John Wiley \& Sons, Inc., Toronto, 1996.

[8] MathWorks, "Symbolic Math Toolbox," https://www.mathworks.com/products/symbolic.html, accessed 9 November 2018.

[9] Borer, N. K., Derlaga, J. M., Deere, K. A., Carter, M. B., Viken, S., Patterson, M. D., Litherland, B. L., Stoll, A. M., "Comparison of Aero-Propulsive Performance Prediction for Distributed Propulsion Configurations," 55th AIAA Aerospace Sciences Meeting, AIAA SciTech Forum, (AIAA 2017-0209), January 2017. doi: 10.2514/6.2017-0209

[10] Advanced Navigation, "Spatial Dual Reference Manual," Version 2.3, December 2016, available at: https://www.advancednavigation.com/product/spatial-dual, accessed 7 May 2019.

[11] Anderson, Jr., J. D., Aircraft Performance and Design, WCB/McGraw-Hill, New York, 1999.

[12] Neter, J., Kutner, M. H., Nachtsheim, C. J., Wasserman, W., Applied Linear Statistic Models, ${ }^{\text {th }}$ Ed., WCB/McGraw-Hill, New York, 1996.

[13] Nicolosi, F., Marco A. D., Vecchia, P. D., "Stability, Flying Qualities and Parameter Estimation of a Twin-Engine CS23/FAR 23 Certified Light Aircraft," AIAA Atmospheric Flight Mechanics Conference, (AIAA 2010-7947), Aug. 2010. doi: $10.2514 / 6.2010-7947$

[14] United States Department of Defense, “Flying Qualities of Piloted Aircraft,” MIL-STD-1797A, January 1990. 